\title{
Symmetrization of Poisson's equation with Neumann boundary conditions
}

\author{
JEFFREY J. LANGFORD
}

\begin{abstract}
In this paper, we compare the solutions of two PDEs with Neumann boundary conditions, one with given initial data and one with cap symmetrized data. We show that the solution with cap symmetrized data is itself cap symmetrized and exhibits larger convex means. As corollaries, we prove comparison results on spheres and hemispheres, and prove a conjecture of B. Kawohl.
\end{abstract}

Mathematics Subject Classification (2010): 35J05 (primary); 35B05 (secondary).

\section{Overview of results}

The history of comparison theorems in elliptic partial differential equations dates to the mid 1970s, when G. Talenti proved his now famous result known as Talenti's theorem [24]. Talenti compared the solutions of two PDEs that impose homogeneous Dirichlet boundary conditions. To be precise, let $0 \leq f \in L^{2}(\Omega)$ where $\Omega \subset \mathbb{R}^{n}$ is a bounded domain, and consider the solution $u$ to the Poisson PDE

$$
\begin{array}{rll}
-\Delta u=f & \text { in } \quad \Omega, \\
u=0 \quad \text { on } & \partial \Omega,
\end{array}
$$

where $\Delta$ is the standard Laplacian operator. Talenti then considered a second PDE defined on a ball $\Omega^{\#}$ with the same volume as $\Omega$. In this second PDE, the input data is obtained by "rearranging" $f$ 's values into a radial function $f^{\#}$, called the Schwarz rearrangement or symmetric decreasing rearrangement, that decreases as the radial variable $r$ increases. After solving the PDE

$$
\begin{array}{rll}
-\Delta v=f^{\#} & \text { in } & \Omega^{\#}, \\
v=0 & \text { on } & \partial \Omega^{\#},
\end{array}
$$

Talenti found that the two solutions $u$ and $v$ are comparable through their decreasing rearrangements, a finding with consequences about $L^{p}$ norms: $\|u\|_{L^{p}(\Omega)} \leq$ 
$\|v\|_{L^{p}\left(\Omega^{\#}\right)}, 1 \leq p \leq \infty$, and oscillation: $\operatorname{osc}_{\Omega} u=\max _{\Omega} u \leq \max _{\Omega^{\#}} v=\operatorname{osc}_{\Omega^{\#}} v$. Although Talenti considered more general elliptic operators for the "initial" PDE, we will concentrate on the Laplace operator.

The work of Talenti sparked a study of comparison theorems using different rearrangements and/or different boundary conditions. In each of the works [1,14], and [12] the authors compare the solutions of two PDEs with homogeneous Dirichlet boundary conditions, one with initial data $f$, and the other with data $f^{\#}$ obtained from $f$ by performing a Steiner symmetrization. Under Steiner symmetrization, $f^{\#}$ is obtained by performing a Schwarz rearrangement on slice functions of $f$. In each of those papers, the conclusions are not as strong as Talenti's, but they still deduce the same $L^{p}$ norm and oscillation inequalities as in Talenti's theorem.

Theorems also appear in the literature comparing the solution of an initial PDE imposing homogeneous Neumann boundary conditions to a coupled system of PDEs taking various forms: in [22], the coupled system of PDEs imposes homogeneous Dirichlet boundary conditions; in [2], the coupled system of PDEs imposes inhomogeneous Neumann and Dirichlet boundary conditions; and in [16], the coupled system of PDEs imposes mixed boundary conditions.

Also relevant are comparison theorems where rearrangements are taken with respect to measures other than Lebesgue measure. For example, in [9] and [13], the authors obtain comparison results by rearranging with respect to Gauss measure. See also [11] and [8]; in the latter, the authors obtain comparison results by rearranging with respect to certain radial functions living in a particular class.

But what appears missing from the literature are comparison theorems imposing Neumann boundary conditions on the first and second PDE, following in the true spirit of Talenti's theorem. In this paper we prove several such results, and our methods are strikingly different from those used in the above literature. Those papers, in general, use methods adapted from Talenti [24] and involve a detailed analysis of a function's level sets together with tools such as the coarea formula. For those papers that involve Neumann boundary conditions, the relative isoperimetric inequality often plays a crucial role. Our methods, on the other hand, heavily rely on the subharmonicity results of A. Baernstein and the maximum principle, and the relative isoperimetric inequality never arises.

In general, we will begin with a PDE of the form

$$
\begin{aligned}
-\Delta u & =f & \text { in } & \Omega, \\
\frac{\partial u}{\partial n} & =0 & \text { on } & \partial \Omega,
\end{aligned}
$$

where $\Omega$ is either a spherical shell, a ball, a sphere, a hemisphere, or a rectangle. Our rearranged PDE will be defined on the same space, with

$$
\begin{aligned}
-\Delta v & =f^{\#} \quad \text { in } \quad & \Omega, \\
\frac{\partial v}{\partial n}=0 & \text { on } & \partial \Omega,
\end{aligned}
$$


where $f^{\#}$ is some rearrangement of $f$. We identify spaces and rearrangements that yield comparison theorems with the same $L^{p}$ norm and oscillation consequences as Talenti's theorem, namely, that $\|u\|_{L^{p}(\Omega)} \leq\|v\|_{L^{p}(\Omega)}, 1 \leq p \leq \infty$, and $\operatorname{osc}_{\Omega} u \leq$ $\operatorname{osc}_{\Omega} v$.

Section 2 presents a dictionary of the various rearrangements, star functions, and subharmonicity results that will be used throughout the paper. The heart of this paper is the main results in Section 3 about cap symmetrization, appearing as Theorem 3.1 and Corollary 3.4. Recall the cap symmetrization of a function $f$ defined on a spherical shell is obtained by fixing an "origin" on the sphere and taking a symmetric decreasing rearrangement on $f$ 's radial slice functions. We shall use the "east pole" $e_{1}$ as the sphere's origin. A precise definition of the cap symmetrization can be found in Section 2. The results of Section 3 combine to give the following theorem.

Theorem (Comparison theorem in spherical shells). Let $A=\left\{x \in \mathbb{R}^{n}: a<\right.$ $|x|<b\}$ be a spherical shell with $0<a<b<\infty$ and let $f \in L^{2}(A)$ with $\int_{A} f \mathrm{~d} x=0$. Assume $u$ and $v$ are weak solutions to

$$
\begin{aligned}
-\Delta u & =f \text { in } A, & -\Delta v & =f^{\#} \text { in } A, \\
\frac{\partial u}{\partial n} & =0 \text { on } \partial A, & \frac{\partial v}{\partial n} & =0 \text { on } \partial A, \\
\int_{A} u \mathrm{~d} x & =0, & \int_{A} v \mathrm{~d} x & =0,
\end{aligned}
$$

where $f^{\#}$ is the cap symmetrization of $f$.

Then for almost every $r \in(a, b)$ and each convex function $\phi: \mathbb{R} \rightarrow \mathbb{R}$ we have

$$
\int_{\mathbb{S}^{n-1}} \phi(u(r \xi)) \mathrm{d} \sigma_{n-1}(\xi) \leq \int_{\mathbb{S}^{n-1}} \phi(v(r \xi)) \mathrm{d} \sigma_{n-1}(\xi) .
$$

Consequently,

$$
\|u\|_{L^{p}(A)} \leq\|v\|_{L^{p}(A)}, \quad 1 \leq p \leq \infty
$$

and

$$
\underset{A}{\operatorname{osc}} u \leq \underset{A}{\operatorname{Oosc}} v
$$

Once the above theorem is proved, we can, with a little more work, prove an analogous result in balls (Theorem 3.3). While this ball result is interesting in its own right, the remainder of the paper deals solely with consequences of the shell results. Section 4 is devoted to consequences of the main results and is divided into two parts. The first part gives comparison results on spheres (Corollary 4.1) and hemispheres (Corollary 4.4) that follow from Theorem 3.1. The spherical comparison result follows from the shell comparison result by a homogeneity extension argument and has the following statement. 
Corollary (Spherical comparison theorem). Let $F \in L^{2}\left(\mathbb{S}^{n}\right)$ with $\int_{\mathbb{S}^{n}} F \mathrm{~d} \sigma_{n}=0$. Assume $U$ and $V$ are weak solutions to

$$
-\Delta_{\mathbb{S}} U=F \text { in } \mathbb{S}^{n}, \quad-\Delta_{\mathbb{S}} V=F^{\#} \text { in } \mathbb{S}^{n},
$$

where $F^{\#}$ is the spherical rearrangement of $F$. Additionally assume $U$ and $V$ are additively normalized so that $\int_{\mathbb{S}^{n}} U \mathrm{~d} \sigma_{n}=\int_{\mathbb{S}^{n}} V \mathrm{~d} \sigma_{n}=0$. Then for every convex function $\phi: \mathbb{R} \rightarrow \mathbb{R}$,

$$
\int_{\mathbb{S}^{n}} \phi(U) \mathrm{d} \sigma_{n} \leq \int_{\mathbb{S}^{n}} \phi(V) \mathrm{d} \sigma_{n} .
$$

Consequently,

$$
\begin{aligned}
\|U\|_{L^{p}\left(\mathbb{S}^{n}\right)} & \leq\|V\|_{L^{p}\left(\mathbb{S}^{n}\right)}, \quad 1 \leq p \leq \infty \\
\text { ess sup } U & \leq \underset{\mathbb{S}^{n}}{\operatorname{ess} \sup V}, \\
\mathbb{S}^{n} & \underset{\mathbb{S}^{n}}{\operatorname{ess} \inf U} \geq \underset{\mathbb{S}^{n}}{\operatorname{ess} \inf V} \\
\underset{\mathbb{S}^{n}}{\operatorname{Osc}} U & \leq \underset{\mathbb{S}^{n}}{\operatorname{osc}} V .
\end{aligned}
$$

Working on manifolds, A. Cianchi [15] obtains a very similar result to the one above, imposing a different normalization on the solutions $U$ and $V$. In Remark 4.2, we show how to obtain Cianchi's result using our methods. The first part of Section 4 ends with a hemispherical comparison result that follows from the spherical result by a reflection, or "gluing", argument. The statement of the hemisphere result is analogous to the spherical one, except that it additionally imposes Neumann boundary conditions on the hemisphere boundary.

The second part of Section 4 contains a proof of a conjecture raised by B. Kawohl [19, page 61]. Kawohl considered a rearrangement called the monotone decreasing rearrangement in the direction $y$ for functions defined in a rectangle. That rearrangement is obtained by holding the first variable fixed and taking the decreasing rearrangement with respect to the second variable. Here is the conjecture.

Conjecture (Kawohl's conjecture). Let $R$ be the unit square $(0,1) \times(0,1)$ in $\mathbb{R}^{2}$ and $f: R \rightarrow \mathbb{R}$ a sufficiently smooth function with mean value zero. Consider the problems:

$$
\begin{aligned}
-\Delta u & =f \text { in } R, & -\Delta v & =f^{\#} \text { in } R, \\
\frac{\partial u}{\partial n} & =0 \text { on } \partial R, & \frac{\partial v}{\partial n} & =0 \quad \text { on } \partial R,
\end{aligned}
$$

where $f^{\#}$ is the monotone decreasing rearrangement of $f$ in the direction $y$.

Then the oscillation of $u$ over $\bar{R}$ should be dominated by the oscillation of $v$. 
In 1986, Baernstein wrote Kawohl with a solution (unpublished) containing three components:

1) an annular comparison result (a two-dimensional version of Theorem 3.1),

2) a rectangular comparison result, and

3) a rephrasing of the rectangular comparison result in terms of convex means.

The third component was then used to prove Kawohl's conjecture. In Section 4, we will follow the same basic structure as Baernstein, but provide different (and simpler) proofs for each step.

The theory of the star function has been further developed since the correspondence, and so the proof of Kawohl's conjecture we present differs from the one originally sent by Baernstein. In Section 4, we show how to obtain a rectangular comparison result from the two-dimensional version of Theorem 3.1. Baernstein indicated to Kawohl that this rectangular comparison result was the key to the conjecture. However, Baernstein did not provide the details of how this rectangular comparison result is obtained, and so we provide them in Section 4 before closing with a proof of Kawohl's conjecture that differs from the one originally sent by Baernstein. Our proof of Kawohl's conjecture does not rely on rephrasing the rectangular comparison result in terms of convex means. Instead, we use the rectangular comparison result directly.

The proof of Kawohl's conjecture presented here is not the first to appear in print. In [10], F. Brock proves Kawohl's conjecture using Green's function representations of the solutions together with a Hardy-Littlewood-type rearrangement inequality. In fact, Brock proves a bit more than Kawohl's conjecture. As mentioned above, we prove Kawohl's conjecture from a rectangular comparison result, which also gives the additional conclusions obtained by Brock, at least in Kawohl's setting.

\section{Background}

\subsection{Rearrangements and star functions for general measure spaces}

Throughout this section, $(X, \mu)$ denotes a fixed measure space.

Definition 2.1 (Rearrangements). Given functions $f \in L^{1}(X)$ and $g \in L^{1}(Y)$ defined on measure spaces $(X, \mu)$ and $(Y, v)$, we say $f$ and $g$ are rearrangements of each other if

$$
\mu(\{x \in X: t<f(x)\})=v(\{y \in Y: t<g(y)\})
$$

for every $t \in \mathbb{R}$.

A good starting point for analysts who want to learn about rearrangement methods is the book by Lieb and Loss [20], which tackles a number of standard and not so standard results in analysis using rearrangement methods.

We next define the decreasing rearrangement. 
Definition 2.2 (Decreasing rearrangement). Let $f \in L^{1}(X)$ and define $f^{*}$ : $[0, \mu(X)] \rightarrow[-\infty, \infty]$ by the formula

$$
f^{*}(t)= \begin{cases}\operatorname{ess} \sup f & \text { if } t=0, \\ X & \text { if } t \in(0, \mu(X)), \\ \inf \{s: \mu(\{x: s<f(x)\}) \leq t\} & \text { if } t=\mu(X) . \\ \underset{X}{\operatorname{exsinf} f} & \end{cases}
$$

We call $f^{*}$ the decreasing rearrangement of $f$.

Note that our definition of the decreasing rearrangement takes into account $f$ 's positive and negative values. Many authors choose to define the decreasing rearrangement as $|f|^{*}$ in our notation.

We now define the notion of a "star function" for a general measure space. Proposition 2.6 below establishes the connection between the star function and the decreasing rearrangement.

Definition 2.3 (Star function for a general measure space). Let $f \in L^{1}(X)$. The star function of $f$ will be denoted by $f^{\star}$ and is defined on the interval $[0, \mu(X)]$ by the formula

$$
f^{\star}(t)=\sup _{\mu(E)=t} \int_{E} f \mathrm{~d} \mu,
$$

where the sup is taken over all measurable subsets $E \subseteq X$ with $\mu(E)=t$.

We now proceed to define those measure spaces for which the sup defining $f^{\star}(t)$ is achieved.

Definition 2.4. Assume $(X, \mu)$ is a measure space and $B \subseteq X$ with $0<\mu(B)$. We say $B$ is an atom if for every subset $A \subseteq B$, either $\mu(A)=\mu(B)$ or $\mu(A)=0$. The measure space $(X, \mu)$ is called non-atomic if it contains no atoms.

A result of W. Sierpiński says that a non-atomic measure space assumes a continuum of values. Precisely, given a subset $B \subseteq X$ with $0<\mu(B)$, for any $a \leq \mu(B)$ there exists a subset of $A \subseteq B$ with $\mu(A)=a$. See [17, Theorem 13].

It is a result of Baernstein that for any $t$ value, there exists a subset $E \subseteq X$ for which the sup defining $f^{\star}(t)$ is achieved [4, Proposition 1]. We record this result below. Baernstein's proof in [4] is for the case when $X$ is an interval and $\mu$ is Lebesgue measure, but his proof carries over for non-atomic measure spaces without change. When we define star functions later on, we are thus justified using max instead of sup.

Proposition 2.5. Assume $f \in L^{1}(X)$ with $(X, \mu)$ a finite non-atomic measure space. Given $t \in[0, \mu(X)]$, there exists a subset $E \subseteq X$ such that

$$
f^{\star}(t)=\int_{E} f \mathrm{~d} \mu .
$$

Thus, the sup defining $f^{\star}$ is really a max. 
The set of length $t$ on which the decreasing rearrangement $f^{*}$ is biggest is the interval $[0, t]$. Since $f^{*}$ is a rearrangement of $f$, it follows that $f^{\star}(t)=$ $\int_{0}^{t} f^{*}(x) \mathrm{d} x$. We record this fact as Proposition 2.6 below, which appears as [4, Proposition 2] in the case where $X$ is an interval and $\mu$ is Lebesgue measure; the proof carries over without change for the case of a general non-atomic measure space.

Proposition 2.6. Assume $f \in L^{1}(X)$ with $(X, \mu)$ a finite non-atomic measure space. Then for each $t \in[0, \mu(X)]$,

$$
f^{\star}(t)=\int_{0}^{t} f^{*}(x) \mathrm{d} x,
$$

where $f^{*}$ is the decreasing rearrangement of $f$.

Star functions first appeared as a tool to prove Edrei's spread conjecture [3] about growth of meromorphic functions in the plane, and have since been used to solve other extremal problems involving various norms of Schlicht functions and Green's functions $[4,5]$.

Star function inequalities define a type of majorization. Our next proposition says that star function inequalities can be rephrased in terms of convex mean inequalities and appears as [4, Proposition 3]. The proof in [4, Proposition 3] is for functions defined on an interval. By passing to decreasing rearrangements, the result also holds for functions defined on a general measure space.

Proposition 2.7 (Majorization). Let $u, v \in L^{1}(X)$ where $(X, \mu)$ is a finite measure space. Then

$$
u^{\star} \leq v^{\star}
$$

on $[0, \mu(X)]$ if and only if the inequality

$$
\int_{X} \phi(u) \mathrm{d} \mu \leq \int_{X} \phi(v) \mathrm{d} \mu
$$

holds for every increasing convex function $\phi: \mathbb{R} \rightarrow \mathbb{R}$.

Moreover, if $\int_{X} u \mathrm{~d} \mu=\int_{X} v \mathrm{~d} \mu$, then the word "increasing" may be removed from the previous statement.

Baernstein's proof in [4, Proposition 3] only gives the first part of Proposition 2.7 above. We supply a proof of the second part below.

Proof. Under the assumptions of Proposition 2.7, assume that $u$ and $v$ share the same mean, and that the inequality

$$
\int_{X} \phi(u) \mathrm{d} \mu \leq \int_{X} \phi(v) \mathrm{d} \mu
$$


holds whenever $\phi$ is an increasing convex function. Let $\phi: \mathbb{R} \rightarrow \mathbb{R}$ be a convex function (not necessarily increasing). We verify that the above inequality still holds.

The one sided derivatives $\phi^{\prime}(x+)$ and $\phi^{\prime}(x-)$ exist at every $x$ and are both increasing functions of $x$. First assume that

$$
\left|\phi^{\prime}(x+)\right| \leq M
$$

for some constant $M$ and let $x_{1}<x_{2}$. Then

$$
-M \leq \phi^{\prime}\left(x_{1}+\right) \leq \frac{\phi\left(x_{2}\right)-\phi\left(x_{1}\right)}{x_{2}-x_{1}}
$$

which implies that

$$
\phi\left(x_{1}\right)+M x_{1} \leq \phi\left(x_{2}\right)+M x_{2} .
$$

That is, $\phi(x)+M x$ is an increasing convex function. Thus,

$$
\int_{X}(\phi(u)+M u) \mathrm{d} \mu \leq \int_{X}(\phi(v)+M v) \mathrm{d} \mu
$$

holds, and since $u$ and $v$ have the same mean, $\int_{X} \phi(u) \mathrm{d} \mu \leq \int_{X} \phi(v) \mathrm{d} \mu$ follows. We next remove the assumption that $\phi^{\prime}(x+)$ is bounded.

Given $\phi$, define

$$
\phi_{n}(x)= \begin{cases}\phi^{\prime}((-n)-)(x+n)+\phi(-n) & \text { if } x \leq-n, \\ \phi(x) & \text { if }-n<x<n, \\ \phi^{\prime}(n+)(x-n)+\phi(n) & \text { if } x \geq n,\end{cases}
$$

where $\phi^{\prime}((-n)-)$ denotes the left sided derivative of $\phi$ at $-n$. Then each $\phi_{n}^{\prime}(x+)$ is bounded. Moreover, $\phi_{n}$ is convex, $\phi_{n} \leq \phi$, and $\phi_{n} \leq \phi_{n+1}$ for every $n$. Let $L$ be a linear function where $L \leq \phi_{1}$. Then

$$
\int_{X}\left(\phi_{n}(u)-L(u)\right) \mathrm{d} \mu \leq \int_{X}\left(\phi_{n}(v)-L(v)\right) \mathrm{d} \mu .
$$

Letting $n \rightarrow \infty$, applying the Monotone convergence theorem, and using the assumption that $u$ and $v$ share the same mean gives the final result $\int_{X} \phi(u) \mathrm{d} \mu \leq$ $\int_{X} \phi(v) \mathrm{d} \mu$.

Two remarks are in order concerning Proposition 2.7.

Remark 2.8. The above proposition does not assert that the phi integrals are finite.

Remark 2.9. Under the assumptions of Proposition 2.7, if $u$ and $v$ have the same mean, then the star function inequality $u^{\star} \leq v^{\star}$ is equivalent to the star function inequalities $u_{+}^{\star} \leq v_{+}^{\star}$ and $u_{-}^{\star} \leq v_{-}^{\star}$ for the positive and negative parts of $u$ and $v$. Hence, all of the comparison results in the sequel have analogous conclusions for the positive and negative parts of the solutions.

Before proceeding we need the following definition. 
Definition 2.10 (Oscillation). If $u: X \rightarrow \mathbb{R}$ is measurable, we define the oscillation by

$$
\underset{X}{\operatorname{osc}} u=\underset{X}{\operatorname{ess} \sup } u-\underset{X}{\operatorname{ess} \inf } u .
$$

The next corollary gives two important consequences of majorization in the sense of star functions.

Corollary 2.11. Let $u, v \in L^{1}(X)$ where $\int_{X} u \mathrm{~d} \mu=\int_{X} v \mathrm{~d} \mu$ and assume $(X, \mu)$ is a finite measure space. If $u^{\star} \leq v^{\star}$ on $[0, \mu(X)]$, then

$$
\|u\|_{L^{p}(X, \mathrm{~d} \mu)} \leq\|v\|_{L^{p}(X, \mathrm{~d} \mu)}, \quad 1 \leq p \leq \infty .
$$

Moreover,

$$
\begin{aligned}
& \text { ess sup } u \leq \text { ess sup } v \text {, } \\
& X \quad X \\
& \underset{X}{\operatorname{ess} \inf } u \geq \underset{X}{\operatorname{ess} \inf } v \text {, } \\
& \underset{X}{\operatorname{osc}} u \leq \underset{X}{\operatorname{osc}} v .
\end{aligned}
$$

Remark 2.12. It is not assumed that the $L^{p}$ norms, ess inf, ess sup, and osc above are finite. Rather, if the $L^{p}$ norm of $v$ is finite, then so is the $L^{p}$ norm of $u$. Likewise, if the $L^{p}$ norm of $u$ is infinite, then so is the $L^{p}$ norm of $v$. Similar considerations apply to the other inequalities.

Proof. By Proposition 2.7, the inequality

$$
\int_{X} \phi(u) \mathrm{d} \mu \leq \int_{X} \phi(v) \mathrm{d} \mu
$$

holds for each convex function $\phi: \mathbb{R} \rightarrow \mathbb{R}$. Taking $\phi(x)=|x|^{p}$ establishes the $L^{p}$ norm inequality for $1 \leq p<\infty$. Letting $p \rightarrow \infty$ gives the case when $p=\infty$.

To establish the ess sup inequality, we rewrite $u^{\star} \leq v^{\star}$ using Proposition 2.6 as

$$
\int_{0}^{t} u^{*}(s) \mathrm{d} s \leq \int_{0}^{t} v^{*}(s) \mathrm{d} s
$$

for every $0 \leq t \leq \mu(X)$. Multiplying the inequality above by $\frac{1}{t}$ and taking the limit as $t \rightarrow 0$, we obtain

$$
\begin{aligned}
\underset{X}{\operatorname{ess} \sup u} & =\operatorname{ess}_{[0, \mu(X)]} u^{*} \\
& =\lim _{t \rightarrow 0} \frac{1}{t} \int_{0}^{t} u^{*}(s) \mathrm{d} s \\
& \leq \lim _{t \rightarrow 0} \frac{1}{t} \int_{0}^{t} v^{*}(s) \mathrm{d} s \\
& =\operatorname{ess~sup}_{[0, \mu(X)]} v^{*} \\
& =\underset{X}{\operatorname{ess} \sup v .}
\end{aligned}
$$


Since $\int_{X} u \mathrm{~d} \mu=\int_{X} v \mathrm{~d} \mu$, we also have $\int_{t}^{\mu(X)} v^{*}(s) \mathrm{d} s \leq \int_{t}^{\mu(X)} u^{*}(s) \mathrm{d} s$ for each $0 \leq t \leq \mu(X)$, and so an argument similar to the one above gives

$$
\underset{X}{\operatorname{ess} \inf } v \leq \underset{X}{\operatorname{ess} \inf } u
$$

The osc inequality now follows by combining the ess sup and ess inf inequalities.

\subsection{Rearrangements and star functions for spheres and spherical shells}

Spheres. The spherical rearrangement gives an analogue of the Schwarz rearrangement, or symmetric decreasing rearrangement, for functions defined on spheres. We write

$$
\mathbb{S}^{n}=\left\{\left(\xi_{1}, \xi_{2}, \ldots, \xi_{n+1}\right) \in \mathbb{R}^{n+1}: \xi_{1}^{2}+\xi_{2}^{2}+\ldots+\xi_{n+1}^{2}=1\right\}
$$

for the unit $n$-sphere in $\mathbb{R}^{n+1}$, and $\sigma_{n}$ for surface measure on $\mathbb{S}^{n}$. So, for example, $\sigma_{1}\left(\mathbb{S}^{1}\right)=2 \pi$. Let $d$ denote the standard distance on $\mathbb{S}^{n}$ whereby the distance between any two points is calculated by computing the length of the shorter arc of the great circle that joins them. We write

$$
K(\theta)=\left\{\xi \in \mathbb{S}^{n}: d\left(\xi, e_{1}\right)<\theta\right\}
$$

for the open polar cap centered at the "east pole" $e_{1}=(1,0, \ldots, 0)$ and of radius $\theta$ (in the spherical distance). For example, when $n=1, K(\theta)=\left\{e^{i \phi}:-\theta<\phi<\theta\right\}$. A function defined on the sphere can be rearranged into one that is constant on boundaries of caps centered at the east pole, and that decreases on these cap boundaries as they sweep out the sphere from $e_{1}$ to $-e_{1}$. The spherical rearrangement thus provides an analogue of the Schwarz rearrangement for the sphere.

Definition 2.13 (Spherical rearrangement). Given $F \in L^{1}\left(\mathbb{S}^{n}\right)$, we define $F^{\#}$ : $\mathbb{S}^{n} \rightarrow[-\infty, \infty]$ by the formula

$$
F^{\#}(\xi)=F^{*}\left(\sigma_{n}(K(\theta))\right),
$$

where $\theta$ is the spherical distance between the point $\xi$ and $e_{1}$, and $F^{*}$ is the decreasing rearrangement of $F$. We call $F^{\#}$ the spherical rearrangement of $F$.

With the spherical rearrangement in hand, we have the following star function definition.

Definition 2.14 (Star functions on spheres). Given $F \in L^{1}\left(\mathbb{S}^{n}\right)$, we define $F^{\star}$ : $(0, \pi) \rightarrow \mathbb{R}$ by the formula

$$
F^{\star}(\theta)=\max _{\sigma_{n}(E)=\sigma_{n}(K(\theta))} \int_{E} F \mathrm{~d} \sigma_{n},
$$

where the max is taken over all measurable subsets $E$ of $\mathbb{S}^{n}$ with the same surface measure as the open cap $K(\theta)$. 
Observe the definition above agrees with Definition 2.3 after making the change of variable $t=\sigma_{n}(K(\theta))$. Just as in Proposition 2.5, the max defining $F \star$ is achieved for some subset $E$, which explains our use of max instead of sup.

The set of surface measure $\sigma_{n}(K(\theta))$ on which $F^{\#}$ is biggest is the polar cap $K(\theta)$. Since $F$ and $F^{\#}$ are rearrangements, it follows just as in Proposition 2.6 that

$$
F^{\star}(\theta)=\int_{K(\theta)} F^{\#} \mathrm{~d} \sigma_{n}
$$

Thus, when $n=1$, we have

$$
F^{\star}(\theta)=\int_{-\theta}^{\theta} F^{\#}\left(e^{i \phi}\right) \mathrm{d} \phi
$$

Spherical shells. Throughout this subsection, $A \subset \mathbb{R}^{n}$ denotes a spherical shell $A=A(a, b)=\left\{x \in \mathbb{R}^{n}: a<|x|<b\right\}$ for real numbers $0 \leq a<b<\infty$. Given a function $f: A \rightarrow \mathbb{R}$, we can spherically rearrange $f$ on each concentric $(n-1)$-sphere. Doing so gives the cap symmetrization.

Definition 2.15 (Cap symmetrization). Given $f \in L^{1}(A)$, we define $f^{\#}: A \rightarrow$ $[-\infty, \infty]$ as follows. By Fubini's theorem, the slice function $f^{r}: \mathbb{S}^{n} \rightarrow \mathbb{R}$ defined by $f^{r}(\xi)=f(r \xi)$ belongs to $L^{1}\left(\mathbb{S}^{n-1}\right)$ for almost every $r \in(a, b)$. For such $r$, we define the cap symmetrization of $f$ on $\{|x|=r\}$ by

$$
f^{\#}(r \xi)=\left(f^{r}\right)^{\#}(\xi),
$$

where $\left(f^{r}\right)^{\#}$ denotes the spherical rearrangement of the slice function $f^{r}$. We leave $f^{\#}$ undefined on those spheres $\{|x|=r\}$ when $f^{r} \notin L^{1}\left(\mathbb{S}^{n-1}\right)$.

Star functions corresponding to cap symmetrization will be defined in a polar rectangle. Write

$$
A^{\star}=\left\{(r, \theta) \in \mathbb{R}^{2}: a<r<b \text { and } 0<\theta<\pi\right\} .
$$

The idea is to take a spherical star function on each slice function $f^{r}$. Since cap symmetrization is a partial symmetrization, meaning rearrangement takes place inside subsets of codimension one, the corresponding star function will be of two variables, $r$ and $\theta$.

Definition 2.16 (Star functions on spherical shells). If $f \in L^{1}(A)$, define $f^{\star}$ : $A^{\star} \rightarrow \mathbb{R}$ a.e. by the formula

$$
f^{\star}(r, \theta)=\max _{\sigma_{n-1}(E)=\sigma_{n-1}(K(\theta))} \int_{E} f(r \xi) \mathrm{d} \sigma_{n-1}(\xi)=\int_{K(\theta)} f^{\#}(r \xi) \mathrm{d} \sigma_{n-1}(\xi)
$$

where the max is taken over all measurable subsets $E$ of $\mathbb{S}^{n-1}$ with the same surface measure as $K(\theta)$ and $f^{\#}$ denotes the cap symmetrization of $f$. 
Note that $f^{\star}(r, \theta)$ is defined for every $\theta$ when the slice function $f^{r} \in L^{1}\left(\mathbb{S}^{n-1}\right)$. Just as in Proposition 2.5, the max defining $f \star$ is achieved for some subset $E$, which explains our use of max rather than sup.

The following result will come in handy later. It says that if a sequence of functions converges in $L^{1}$, then by passing to a subsequence we have almost everywhere pointwise convergence for the star functions involved.

Proposition 2.17 (Convergence of star functions). Assume $u, u_{k} \in L^{1}(A)$ and $u_{k} \rightarrow u$ in $L^{1}(A)$. Then for some subsequence and for almost every $r \in(a, b)$, we have

$$
\int_{\mathbb{S}^{n-1}}\left|u_{k_{j}}(r \xi)-u(r \xi)\right| \mathrm{d} \sigma_{n-1}(\xi) \rightarrow 0
$$

and consequently

$$
u_{k_{j}}^{\star}(r, \theta) \rightarrow u^{\star}(r, \theta)
$$

for every $\theta \in(0, \pi)$. In particular, $u_{k_{j}}^{\star} \rightarrow u^{\star}$ a.e. in $A^{\star}$.

Proof. Define $\Psi_{k}:(a, b) \rightarrow \mathbb{R}$ by the formula

$$
\Psi_{k}(r)=\int_{\mathbb{S}^{n-1}}\left|u_{k}(r \xi)-u(r \xi)\right| \mathrm{d} \sigma_{n-1}(\xi) .
$$

By assumption, $u_{k} \rightarrow u$ in $L^{1}(A)$. That is,

$$
\int_{a}^{b} \int_{\mathbb{S}^{n-1}}\left|u_{k}(r \xi)-u(r \xi)\right| \mathrm{d} \sigma_{n-1}(\xi) r^{n-1} \mathrm{~d} r \rightarrow 0,
$$

which implies that $\Psi_{k} \rightarrow 0$ in $L^{1}\left((a, b), r^{n-1} \mathrm{~d} r\right)$. Thus, we can pass to a subsequence where $\Psi_{k_{j}} \rightarrow 0$ a.e. in $(a, b)$. By the very definition of $\Psi_{k_{j}}$, this implies

$$
\int_{\mathbb{S}^{n-1}}\left|u_{k_{j}}(r \xi)-u(r \xi)\right| \mathrm{d} \sigma_{n-1}(\xi) \rightarrow 0
$$

for almost every $r \in(a, b)$, which gives the first conclusion. Fix an $r$ so that convergence holds above. Then for any $\theta \in(0, \pi)$ we have

$$
\begin{aligned}
\left|u_{k_{j}}^{\star}(r, \theta)-u^{\star}(r, \theta)\right| & =\left|\int_{K(\theta)} u_{k_{j}}^{\#}(r \xi)-u^{\#}(r \xi) \mathrm{d} \sigma_{n-1}(\xi)\right| \\
& \leq \int_{\mathbb{S}^{n-1}}\left|u_{k_{j}}^{\#}(r \xi)-u^{\#}(r \xi)\right| \mathrm{d} \sigma_{n-1}(\xi) \\
& \leq \int_{\mathbb{S}^{n-1}}\left|u_{k_{j}}(r \xi)-u(r \xi)\right| \mathrm{d} \sigma_{n-1}(\xi),
\end{aligned}
$$

where the last inequality holds because the spherical rearrangement is a contraction in the $L^{1}$ distance (see [6, Theorem 3], for example). Letting $j \rightarrow \infty$, we conclude

$$
u_{k_{j}}^{\star}(r, \theta) \rightarrow u^{\star}(r, \theta)
$$




\subsection{Commutativity and subharmonicity results in shells}

We now state "commutativity" and "subharmonicity" results for cap symmetrization that require some notation. Let $A=A(a, b)=\left\{x \in \mathbb{R}^{n}: a<|x|<b\right\}$ denote a spherical shell as before with inner radius $0 \leq a$ and outer radius $b<\infty$ and recall the notation $A^{\star}=(a, b) \times(0, \pi)$. Given $u \in L^{1}(A)$, we define $J u: A^{\star} \rightarrow \mathbb{R}$ a.e. by

$$
J u(r, \theta)=\int_{K(\theta)} u(r \xi) \mathrm{d} \sigma_{n-1}(\xi) .
$$

With this notation, we have

$$
u^{\star}(r, \theta)=\int_{K(\theta)} u^{\#}(r \xi) \mathrm{d} \sigma_{n-1}(\xi)=J u^{\#}(r, \theta)
$$

where $u^{\#}$ denotes the cap symmetrization of $u$.

We let $\Delta$ denote the standard Laplacian operator in $\mathbb{R}^{n}$ and define new operators $\Delta^{\star}$ and $\Delta^{\star}$ which act on functions $F \in C^{2}\left(A^{\star}\right)$ as follows:

$$
\begin{aligned}
\Delta^{\star} F= & \partial_{r r} F+\frac{n-1}{r} \partial_{r} F+r^{-2}\left[\partial_{\theta \theta} F-(n-2)(\cot \theta) \partial_{\theta} F\right], \\
\Delta^{\star t} F= & \partial_{r r} F-\frac{n-1}{r} \partial_{r} F+r^{-2}(n-1) F \\
& +r^{-2}\left[\partial_{\theta \theta} F+(n-2)(\cot \theta) \partial_{\theta} F-(n-2)\left(\csc ^{2} \theta\right) F\right] .
\end{aligned}
$$

The $\Delta^{\star t}$ operator is the formal adjoint of $\Delta^{\star}$ with respect to the standard inner product on $L^{2}\left(A^{\star}, \mathrm{d} r \mathrm{~d} \theta\right)$.

Theorem 2.18 and 2.20 below appear as equation (5.9) and [6, Theorem 5], respectively.

Theorem 2.18 (Commutativity relation for cap symmetrization). If $u \in C^{2}(A)$, then

$$
J \Delta u=\Delta^{\star} J u
$$

on $A^{\star}$.

Theorem 2.18 is proved by using polar coordinates together with the decomposition $\Delta=\frac{\partial^{2}}{\partial r^{2}}+\frac{n-1}{r} \frac{\partial}{\partial r}+\frac{1}{r^{2}} \Delta_{\mathbb{S}}$, where $\Delta_{\mathbb{S}}$ is the spherical Laplacian.

Before proceeding, we make the following definition.

Definition 2.19. For $u \in C^{2}(A)$ and $f \in L_{l o c}^{1}(A)$, we say that $-\Delta^{\star} u^{\star} \leq f^{\star}$ in the weak sense provided the inequality

$$
-\int_{A^{\star}} u^{\star} \Delta^{\star t} G \mathrm{~d} r \mathrm{~d} \theta \leq \int_{A^{\star}} f^{\star} G \mathrm{~d} r \mathrm{~d} \theta
$$

holds for every nonnegative $G \in C_{c}^{2}\left(A^{\star}\right)$. 
Theorem 2.20 (Subharmonicity for cap symmetrization). Suppose $u \in C^{2}(A)$ satisfies $-\Delta u=f$. Then

$$
-\Delta^{\star} u^{\star} \leq f^{\star}
$$

in the weak sense.

A new proof of Theorem 2.20 appears in the Appendix. The proof is due to Baernstein and is much simpler than the proof of a more general result (Theorem 5) that appears in [6].

The two-dimensional case. In dimension $n=2$ it is helpful to use complex notation. In this case, $A=A(a, b)=\{z \in \mathbb{C}: a<|z|<b\}$ and we will write $A^{\star}=\{z \in A: \operatorname{Im}(z)>0\}$. So if $u \in L^{1}(A)$,

$$
J u\left(r e^{i \theta}\right)=\int_{-\theta}^{\theta} u\left(r e^{i \phi}\right) \mathrm{d} \phi,
$$

and for the star function $u^{\star}: A^{\star} \rightarrow \mathbb{R}$ we write

$$
u^{\star}\left(r e^{i \theta}\right)=\max _{|E|=2 \theta} \int_{E} u\left(r e^{i \phi}\right) \mathrm{d} \phi .
$$

Then $u^{\star}=J u^{\#}$ where $u^{\#}$ is the cap symmetrization of $u$. The following commutativity result, which appears as [7, Proposition 3.1], is a special case of Theorem 2.18. Note that in dimension $n=2$, the star operator $\Delta^{\star}$ equals the standard planar Laplacian $\Delta$ expressed in polar coordinates.

Theorem 2.21 (Commutativity relation for cap symmetrization). Let $u \in C^{2}(A)$. Then

$$
\Delta J u=J \Delta u
$$

on $A^{\star}$.

The following subharmonicity result is a special case of Theorem 2.20.

Theorem 2.22 (Subharmonicity for cap symmetrization). Suppose $u \in C^{2}(A)$ with $-\Delta u=f$ in $A$. Then

$$
-\Delta u^{\star} \leq f^{\star}
$$

in the weak sense.

Theorems 2.21 and 2.22 explain the reason for the labels "commutativity" and "subharmonicity". When the dimension $n=2$, the $J$ operator commutes with the Laplacian. Additionally, if $u$ is harmonic in an annulus, then the star function $u \star$ is subharmonic in the upper annulus $A^{\star}$. 


\section{Main results}

In this section we present the main results of this paper. In Theorems 3.1 and 3.3, we compare the solutions of two PDEs, one with given initial data and one with cap symmetrized data. We see that the solution with cap symmetrized data has a larger star function. As a corollary, the solution with cap symmetrized data has larger $L^{p}$ norms and oscillation (Corollary 3.4). Additionally, when the input data is cap symmetrized, so is the solution (Corollary 3.5).

Theorem 3.1 (Comparison theorem in spherical shells). Let $A=A(a, b) \subset \mathbb{R}^{n}$ be a spherical shell with $0<a<b<\infty$ and let $f \in L^{2}(A)$ with $\int_{A} f \mathrm{~d} x=0$. Assume $u$ and $v$ are weak solutions to

$$
\begin{array}{rlrl}
-\Delta u & =f \text { in } A, \quad-\Delta v & =f^{\#} \text { in } A, \\
\frac{\partial u}{\partial n} & =0 \text { on } \partial A, & \frac{\partial v}{\partial n} & =0 \quad \text { on } \partial A,
\end{array}
$$

where $f^{\#}$ denotes the cap symmetrization of $f$.

If the solutions $u$ and $v$ are additively normalized so that $\int_{A} u \mathrm{~d} x=\int_{A} v \mathrm{~d} x=$ 0 , then for almost every $r \in(a, b)$,

$$
\int_{\mathbb{S}^{n-1}} u(r \xi) \mathrm{d} \sigma_{n-1}(\xi)=\int_{\mathbb{S}^{n-1}} v(r \xi) \mathrm{d} \sigma_{n-1}(\xi)
$$

and the inequality

$$
u^{\star}(r, \theta) \leq v^{\star}(r, \theta)
$$

holds for every $\theta \in(0, \pi)$. In particular, $u^{\star} \leq v^{\star}$ a.e. in $A^{\star}$.

Proof. First suppose that $f$ is Lipschitz continuous on $\bar{A}$. Since cap symmetrization decreases the modulus of continuity, $f^{\#}$ is also Lipschitz continuous on $\bar{A}$ (this follows from [6, proof of Theorem 3]). Consequently, $u$ and $v$ belong to $C^{2}(\bar{A})[23$, Theorem 3.2].

Step 1: maximum principle. Let

$$
Q(r, \theta)=(r-a)(r-b)+C \theta(\pi-\theta), \quad \text { for } \quad(r, \theta) \in A^{\star},
$$

where $C$ is chosen sufficiently large so that

$$
\Delta^{\star} Q=2+\frac{n-1}{r}(2 r-a-b)-\frac{C}{r^{2}}(2+(n-2) \cot \theta(\pi-2 \theta)) \leq 0 .
$$

Note that

$$
Q_{r}(r, \theta)=2\left(r-\frac{a+b}{2}\right)
$$


and so it follows that

$$
0<\frac{\partial Q}{\partial n} \quad \text { on } \quad(\{a\} \times(0, \pi)) \cup(\{b\} \times(0, \pi)) .
$$

Multiplying $Q$ by a suitable positive constant, we may assume that

$$
\|Q\|_{L^{\infty}\left(A^{\star}\right)} \leq 1
$$

Define

$$
w_{\epsilon}=u^{\star}-J v-\epsilon Q \quad \text { for }(r, \theta) \in A^{\star} \text {. }
$$

In the distributional sense, we compute

$$
\begin{aligned}
-\Delta^{\star} w_{\epsilon} & =-\Delta^{\star} u^{\star}+\Delta^{\star} J v+\epsilon \Delta^{\star} Q \\
& \leq f^{\star}+J \Delta v+0 \\
& =f^{\star}-f^{\star} \\
& =0,
\end{aligned}
$$

where the inequality follows from the subharmonicity and commutativity properties (Theorems 2.18 and 2.20). By the maximum principle applied to $\Delta^{\star}$ [21, Theorem 3]),

$$
\sup _{A^{\star}} w_{\epsilon} \leq \max _{\partial A^{\star}} w_{\epsilon}
$$

Step 2: Boundary analysis. We claim that the max over the boundary cannot be attained at a point of $(\{a\} \times(0, \pi)) \cup(\{b\} \times(0, \pi))$. We prove this by cases.

Case 1: Fix $\left(a, \theta_{1}\right)$ with $\theta_{1} \in(0, \pi)$ and let $E\left(a, K\left(\theta_{1}\right)\right)$ denote a subset of $\mathbb{S}^{n-1}$ with the same surface measure as $K\left(\theta_{1}\right)$ for which the max defining $u^{\star}\left(a, \theta_{1}\right)$ is achieved. We compute for $h>0$

$$
\begin{aligned}
\frac{w_{\epsilon}\left(a+h, \theta_{1}\right)-w_{\epsilon}\left(a, \theta_{1}\right)}{h} \geq & \int_{E\left(a, K\left(\theta_{1}\right)\right)} \frac{u((a+h) \xi)-u(a \xi)}{h} \mathrm{~d} \sigma_{n-1}(\xi) \\
& -\int_{K\left(\theta_{1}\right)} \frac{[v((a+h) \xi)-v(a \xi)]}{h} \mathrm{~d} \sigma_{n-1}(\xi) \\
& -\epsilon \frac{\left[Q\left(a+h, \theta_{1}\right)-Q\left(a, \theta_{1}\right)\right]}{h} .
\end{aligned}
$$

Taking the $\liminf _{h \rightarrow 0}$ and using that $\frac{\partial u}{\partial n}$ and $\frac{\partial v}{\partial n}$ vanish, we get

$$
\liminf _{h \rightarrow 0} \frac{w_{\epsilon}\left(a+h, \theta_{1}\right)-w_{\epsilon}\left(a, \theta_{1}\right)}{h} \geq \epsilon \kappa,
$$


where $\kappa=\frac{\partial Q}{\partial n}\left(a, \theta_{1}\right)$ is some positive number by (3.1). Hence, for all $h>0$ sufficiently small, we have

$$
\begin{aligned}
w_{\epsilon}\left(a+h, \theta_{1}\right) & >w_{\epsilon}\left(a, \theta_{1}\right)+h \frac{\epsilon}{2} \kappa \\
& >w_{\epsilon}\left(a, \theta_{1}\right) .
\end{aligned}
$$

Thus, the maximum of $w_{\epsilon}$ over $\partial A^{\star}$ does not occur at $\left(a, \theta_{1}\right)$, since otherwise the maximum principle (3.2) would be violated.

Case 2: Similar to Case 1, we find the maximum of $w_{\epsilon}$ over $\partial A^{\star}$ does not occur at $\left(b, \theta_{2}\right)$ with $\theta_{2} \in(0, \pi)$.

Our casework above shows that the portion $(\{a\} \times(0, \pi)) \cup(\{b\} \times(0, \pi))$ of $\partial A^{\star}$ may be removed in inequality (3.2). If we write $T=([a, b] \times\{0\}) \cup([a, b] \times$ $\{\pi\})$ then inequality (3.2) becomes

$$
\sup _{A^{\star}} w_{\epsilon} \leq \max _{T} w_{\epsilon}
$$

from which we deduce

$$
\begin{aligned}
-\epsilon+\sup _{A^{\star}}\left(u^{\star}-J v\right) & \leq \sup _{A^{\star}} w_{\epsilon} \\
& \leq \max _{T} w_{\epsilon} \\
& \leq \max _{T}\left(u^{\star}-J v\right)+\epsilon,
\end{aligned}
$$

where the first and last inequalities hold because $|Q| \leq 1$. Letting $\epsilon \rightarrow 0$ we conclude

$$
\sup _{A^{\star}}\left(u^{\star}-J v\right) \leq \max _{T}\left(u^{\star}-J v\right) \text {. }
$$

By definition, $u^{\star}-J v=0$ on $[a, b] \times\{0\}$, that is, when $\theta=0$. We claim $u^{\star}-$ $J v=0$ on $[a, b] \times\{\pi\}$ too. In other words, we claim that $u$ and $v$ have the same integral over each sphere of radius $r \in(a, b)$. Let $\Phi(r)=r^{n-1} \frac{\partial}{\partial r}\left(\int_{\mathbb{S}^{n-1}}(u(r \xi)-\right.$ $\left.v(r \xi)) \mathrm{d} \sigma_{n-1}(\xi)\right)=\int_{\{|x|=r\}}\left(u_{r}-v_{r}\right) \mathrm{d} S$. Then since $\frac{\partial u}{\partial n}$ and $\frac{\partial v}{\partial n}$ are zero when $r=a$, we compute from Green's theorem that

$$
\begin{aligned}
\Phi(r) & =\int_{A(a, r)}(\Delta u-\Delta v) \mathrm{d} x \\
& =\int_{A(a, r)}\left(-f+f^{\#}\right) \mathrm{d} x \\
& =0 .
\end{aligned}
$$

Thus, $\Phi \equiv 0$ on $[a, b]$. By the definition of $\Phi$, this implies that $\int_{\mathbb{S}^{n-1}}(u(r \xi)-$ $v(r \xi)) \mathrm{d} \sigma_{n-1}(\xi)=c$ is constant on $[a, b]$. 
And since $\int_{a}^{b} \int_{\mathbb{S}^{n-1}}(u(r \xi)-v(r \xi)) \mathrm{d} \sigma_{n-1}(\xi) r^{n-1} \mathrm{~d} r=\int_{A}(u-v) \mathrm{d} x=0$ we conclude $c=0$. Hence $\max _{\partial A^{\star}}\left(u^{\star}-J v\right)=0$ so that $u^{\star}-J v \leq 0$ by (3.2). Thus, $u^{\star} \leq J v \leq v^{\star}$, giving the theorem in the case where $f$ is Lipschitz continuous on $\bar{A}$.

Step 3: Approximation argument for general $f$. Now let $f \in L^{2}(A)$ be a general function with mean zero. Choose a sequence of compactly supported smooth functions $f_{k} \in C_{c}^{\infty}(A)$ each with mean zero and where $f_{k} \rightarrow f$ in $L^{2}(A)$. Assume $u_{k}$ and $v_{k}$ solve

$$
\begin{array}{rlrl}
-\Delta u_{k} & =f_{k} \text { in } A, \quad-\Delta v_{k}=f_{k}^{\#} \text { in } A, \\
\frac{\partial u_{k}}{\partial n} & =0 \quad \text { on } \partial A, & \frac{\partial v_{k}}{\partial n} & =0 \quad \text { on } \partial A,
\end{array}
$$

where the $u_{k}$ and $v_{k}$ are normalized so that $\int_{A} u_{k} \mathrm{~d} x=\int_{A} v_{k} \mathrm{~d} x=0$. Since each $f_{k}$ is Lipschitz continuous on $\bar{A}$, our work above shows

$$
\int_{\mathbb{S}^{n-1}} u_{k}(r \xi) \mathrm{d} \sigma_{n-1}(\xi)=\int_{\mathbb{S}^{n-1}} v_{k}(r \xi) \mathrm{d} \sigma_{n-1}(\xi)
$$

for every $r \in(a, b)$ and

$$
u_{k}^{\star} \leq v_{k}^{\star}
$$

in $A^{\star}$ for every $k$. Since cap symmetrization is a contraction in the $L^{2}$ distance [6, Theorem 3], $f_{k}^{\#} \rightarrow f^{\#}$ in $L^{2}(A)$ since $f_{k} \rightarrow f$ in $L^{2}(A)$. Consequently, $u_{k} \rightarrow u$ and $v_{k} \rightarrow v$ in $L^{2}(A)$, essentially a consequence of the Poincaré inequality and Riesz representation theorem. By Proposition 2.17, we can pass to a subsequence of the original $f_{k}$ and assume that for almost every $r \in(a, b)$,

$$
\int_{\mathbb{S}^{n-1}} u_{k}(r \xi) \mathrm{d} \sigma_{n-1}(\xi) \rightarrow \int_{\mathbb{S}^{n-1}} u(r \xi) \mathrm{d} \sigma_{n-1}(\xi)
$$

and that

$$
u_{k}^{\star}(r, \theta) \rightarrow u^{\star}(r, \theta)
$$

for every $\theta \in(0, \pi)$. By another application of Proposition 2.17 and passing to yet another subsequence of the $f_{k}$, we may additionally assume that for almost every $r \in(a, b)$,

$$
\int_{\mathbb{S}^{n-1}} v_{k}(r \xi) \mathrm{d} \sigma_{n-1}(\xi) \rightarrow \int_{\mathbb{S}^{n-1}} v(r \xi) \mathrm{d} \sigma_{n-1}(\xi)
$$

and that

$$
v_{k}^{\star}(r, \theta) \rightarrow v^{\star}(r, \theta)
$$


for every $\theta \in(0, \pi)$. Letting $k \rightarrow \infty$ in (3.4) and (3.5), we therefore conclude that for almost every $r \in(a, b)$,

$$
\int_{\mathbb{S}^{n-1}} u(r \xi) \mathrm{d} \sigma_{n-1}(\xi)=\int_{\mathbb{S}^{n-1}} v(r \xi) \mathrm{d} \sigma_{n-1}(\xi)
$$

and

$$
u^{\star}(r, \theta) \leq v^{\star}(r, \theta)
$$

for every $\theta \in(0, \pi)$.

Remark 3.2. We obtain the same conclusion $u^{\star} \leq v^{\star}$ in Theorem 3.1 if we make the weaker assumption that $u$ and $v$ have the same mean over the shell $A$, not necessarily that they both have zero mean. Moreover, the slice functions of $u$ and $v$ still have the same mean almost everywhere under this weaker assumption.

The analogue of Theorem 3.1 also holds in balls, as we proceed to discuss. Given a ball $B=\left\{x \in \mathbb{R}^{n}:|x|<b\right\}$ with $0<b<\infty$, we define the cap symmetrization of a function $f \in L^{1}(B)$ as follows. When $0<r<b$ and the slice function $f^{r}: \mathbb{S}^{n-1} \rightarrow \mathbb{R}$ belongs to $L^{1}\left(\mathbb{S}^{n-1}\right)$, we define

$$
f^{\#}(r \xi)=\left(f^{r}\right)^{\#}(\xi),
$$

where $\left(f^{r}\right)^{\#}$ is the spherical rearrangement of the slice function $f^{r}$. We leave $f^{\#}$ undefined on the sphere $\{|x|=r\}$ when $f^{r} \notin L^{1}\left(\mathbb{S}^{n-1}\right)$. If $f(0)$ is defined, we define $f^{\#}(0)=f(0)$. And if $f(0)$ is not defined, $f^{\#}(0)$ is also left undefined. The star function $f^{\star}$ will be defined on $B^{\star}=(0, b) \times(0, \pi)$ and will follow Definition 2.16 with $a=0$.

Theorem 3.3 (Comparison theorem balls). Let $B=\left\{x \in \mathbb{R}^{n}:|x|<b\right\}$ where $0<b<\infty$ and say $f \in L^{2}(B)$ with $\int_{B} f \mathrm{~d} x=0$. Assume $u$ and $v$ are weak solutions to

$$
\begin{array}{rlrl}
-\Delta u & =f \text { in } B, \quad-\Delta v & =f^{\#} \text { in } B, \\
\frac{\partial u}{\partial n} & =0 \text { on } \partial B, & \frac{\partial v}{\partial n} & =0 \quad \text { on } \partial B,
\end{array}
$$

where $f^{\#}$ denotes the cap symmetrization of $f$.

If the solutions $u$ and $v$ are additively normalized so that $\int_{B} u \mathrm{~d} x=\int_{B} v \mathrm{~d} x=$ 0 , then for almost every $r \in(0, b)$,

$$
\int_{\mathbb{S}^{n-1}} u(r \xi) \mathrm{d} \sigma_{n-1}(\xi)=\int_{\mathbb{S}^{n-1}} v(r \xi) \mathrm{d} \sigma_{n-1}(\xi)
$$

and the inequality

$$
u^{\star}(r, \theta) \leq v^{\star}(r, \theta)
$$

holds for every $\theta \in(0, \pi)$. In particular, $u^{\star} \leq v^{\star}$ a.e. in $B^{\star}=(0, b) \times(0, \pi)$. 


\section{Proof.}

Step 1: Maximum principle. As before, we assume that $f$ is Lipschitz continuous on $\bar{B}$, which guarantees $u$ and $v$ belong to $C^{2}(\bar{B})$. Let $w_{\epsilon}$ and $Q$ be as in the proof of Theorem 3.1 with $a=0$. If we fix $\epsilon>0$, then $-\Delta u=f$ and $-\Delta v=f^{\#}$ in $A(\epsilon, b)$. Write $A_{\epsilon}^{\star}=(\epsilon, b) \times(0, \pi)$. Then, as before, we have $-\Delta^{\star} w_{\epsilon} \leq 0$ in $A_{\epsilon}^{\star}$. Since the operator $\Delta^{\star}$ is uniformly elliptic in $A_{\epsilon}^{\star}$, we can apply Littman's maximum principle of [21, Theorem 3$]$ ) to conclude

$$
\sup _{A_{\epsilon}^{\star}} w_{\epsilon} \leq \max _{\partial A_{\epsilon}^{\star}} w_{\epsilon}
$$

Since $|Q| \leq 1$, the above inequality implies

$$
-\epsilon+\sup _{A_{\epsilon}^{\star}}\left(u^{\star}-J v\right) \leq \max _{\partial A_{\epsilon}^{\star}}\left(u^{\star}-J v\right)+\epsilon
$$

and letting $\epsilon \rightarrow 0$, we get

$$
\sup _{B^{\star}}\left(u^{\star}-J v\right) \leq \max _{\partial B^{\star}}\left(u^{\star}-J v\right),
$$

where $B^{\star}=(0, b) \times(0, \pi)$.

Step 2: Boundary analysis. As in the proof of Theorem 3.1, we show $u^{\star}-J v \leq 0$ on $\partial B^{\star}$. By definition, $u^{\star}-J v=0$ on $(0, b) \times\{0\}$. The work at the end of Step 2 in the proof of Theorem 3.1 shows that $u$ and $v$ have the same mean over each sphere $\{|x|=r\}$. Hence $u^{\star}-J v=0$ on $(0, b) \times\{\pi\}$, and $u$ and $v$ have the same mean over each ball $\{|x|<r\}$ for $0<r<b$. Letting $r \rightarrow 0$, we conclude $u(0)=v(0)$ so that $u^{\star}(0, \theta)-J v(0, \theta)=(u(0)-v(0)) \sigma_{n-1}(K(\theta))=0$. Hence $u^{\star}-J v$ vanishes on $\{0\} \times(0, \pi)$. Since $\frac{\partial u}{\partial n}$ and $\frac{\partial v}{\partial n}$ vanish on $\{|x|=b\}$, Case 2 of Step 2 in the proof of Theorem 3.1 carries through without change, and shows that $\max _{\partial B \star}\left(u^{\star}-J v\right)$ cannot be attained by a point on $\{b\} \times(0, \pi)$. Thus, $u^{\star}-J v \leq 0$ on $\partial B^{\star}$ so that $u^{\star} \leq J v \leq v^{\star}$ by (3.6).

Step 3: Approximation argument for general $f$. The approximation argument used in the proof of Theorem 3.1 carries over without change to give the result for general $f \in L^{2}(B)$.

The corollary below restates the conclusion of Theorem 3.1 in terms of convex means. This characterization goes all the way back to Hardy, Littlewood, and Pólya [18]. Consequently, we obtain $L^{p}$ and oscillation estimates.

Corollary 3.4. Let $f, u$, and $v$ be as in Theorem 3.1. Then for almost every $r \in$ $(a, b)$ and each convex function $\phi: \mathbb{R} \rightarrow \mathbb{R}$ we have

$$
\int_{\mathbb{S}^{n-1}} \phi(u(r \xi)) \mathrm{d} \sigma_{n-1}(\xi) \leq \int_{\mathbb{S}^{n-1}} \phi(v(r \xi)) \mathrm{d} \sigma_{n-1}(\xi) .
$$


Hence,

$$
\begin{aligned}
\|u(r \cdot)\|_{L^{p}\left(\mathbb{S}^{n-1}\right)} & \leq\|v(r \cdot)\|_{L^{p}\left(\mathbb{S}^{n-1}\right)}, \quad 1 \leq p \leq \infty, \\
\operatorname{ess} \sup u & \leq \operatorname{ess} \sup v \\
|x|=r & \quad|x|=r \\
\operatorname{essinf} u & \geq \underset{|x|=r}{\operatorname{ess} \inf } v \\
|x|=r & \leq \underset{|x|=r}{\operatorname{Oosc}} v .
\end{aligned}
$$

Consequently, for each convex function $\phi: \mathbb{R} \rightarrow \mathbb{R}$,

$$
\int_{A} \phi(u) \mathrm{d} x \leq \int_{A} \phi(v) \mathrm{d} x .
$$

Moreover,

$$
\begin{aligned}
& \|u\|_{L^{p}(A)} \leq\|v\|_{L^{p}(A)}, \quad 1 \leq p \leq \infty, \\
& \text { ess } \sup u \leq \operatorname{ess} \sup v \text {, } \\
& A \quad A \\
& \underset{A}{\operatorname{ess} \inf } u \geq \underset{A}{\operatorname{ess} \inf } v \text {, } \\
& \underset{A}{\operatorname{Osc}} u \leq \underset{A}{\operatorname{osc}} v \text {. }
\end{aligned}
$$

Proof. By Theorem 3.1, for almost every $r \in(a, b), u$ and $v$ have the same mean over the sphere $\{|x|=r\}$ and the inequality $u^{\star}(r, \theta) \leq v^{\star}(r, \theta)$ holds for every $\theta \in(0, \pi)$. Hence, Proposition 2.7 gives

$$
\int_{\mathbb{S}^{n-1}} \phi(u(r \xi)) \mathrm{d} \sigma_{n-1}(\xi) \leq \int_{\mathbb{S}^{n-1}} \phi(v(r \xi)) \mathrm{d} \sigma_{n-1}(\xi)
$$

for each convex function $\phi: \mathbb{R} \rightarrow \mathbb{R}$. The remaining spherical inequalities now follow from Corollary 2.11. The spherical shell inequalities follow from the spherical ones in obvious fashion.

The next corollary tells us that the solution $v$ to the symmetrized problem is cap symmetrized.

Corollary 3.5. If $f$ and $v$ are as in Theorem 3.1, then $v=v^{\#}$ a.e.

Proof. First assume $f$ is Lipschitz continuous on $\bar{A}$. Taking $v=u$, Step 2 in the proof of Theorem 3.1 shows $v^{\star} \leq J v$. Since $J v \leq v^{\star}$ by definition, we have $v^{\star}=J v$ on $A^{\star}$. Fix $r \in(a, b)$. To show $v=v^{\#}$, we first claim that the slice function $v^{r}$ is constant on $\partial K(\theta)$ for each $\theta \in(0, \pi)$. Assume that claim is false. Choose $\theta_{0} \in(0, \pi)$ with $v^{r}$ non-constant on $\partial K\left(\theta_{0}\right)$. Let $\xi_{1}, \xi_{2} \in \partial K\left(\theta_{0}\right)$ be such that

$$
\begin{aligned}
& \min _{\partial K\left(\theta_{0}\right)} v^{r}=v^{r}\left(\xi_{1}\right), \\
& \max _{\partial K\left(\theta_{0}\right)} v^{r}=v^{r}\left(\xi_{2}\right) .
\end{aligned}
$$


Let $\epsilon_{1}, \epsilon_{2}>0$ be small enough so that the spherical balls $B\left(\xi_{1}, \epsilon_{1}\right)$ and $B\left(\xi_{2}, \epsilon_{2}\right)$ are disjoint and

$$
\sup _{B\left(\xi_{1}, \epsilon_{1}\right)} v^{r}<\inf _{B\left(\xi_{2}, \epsilon_{2}\right)} v^{r} .
$$

Additionally, assume $\epsilon_{1}$ and $\epsilon_{2}$ are such that $\left(K\left(\theta_{0}\right) \cup B\left(\xi_{2}, \epsilon_{2}\right)\right) \backslash B\left(\xi_{1}, \epsilon_{1}\right)$ has the same surface measure as $K\left(\theta_{0}\right)$. Geometrically, $\left(K\left(\theta_{0}\right) \cup B\left(\xi_{2}, \epsilon_{2}\right)\right) \backslash B\left(\xi_{1}, \epsilon_{1}\right)$ is constructed from $K\left(\theta_{0}\right)$ by replacing the portion of $B\left(\xi_{1}, \epsilon_{1}\right)$ contained in $K\left(\theta_{0}\right)$ by the portion of $B\left(\xi_{2}, \epsilon_{2}\right)$ contained inside $\mathbb{S}^{n-1} \backslash K\left(\theta_{0}\right)$. Since $v^{\star}=J v$, we have by definition

$$
\int_{E} v^{r} \mathrm{~d} \sigma_{n-1} \leq v^{\star}\left(r, \theta_{0}\right)=\int_{K\left(\theta_{0}\right)} v^{r} \mathrm{~d} \sigma_{n-1}
$$

for all measurable subsets $E \subseteq \mathbb{S}^{n-1}$ with the same surface measure as $K\left(\theta_{0}\right)$. Take $E=\left(K\left(\theta_{0}\right) \cup B\left(\xi_{2}, \epsilon_{2}\right)\right) \backslash B\left(\xi_{1}, \epsilon_{1}\right)$. We compute

$$
\begin{aligned}
\int_{E} v^{r} \mathrm{~d} \sigma_{n-1} & =\int_{K\left(\theta_{0}\right)} v^{r} \mathrm{~d} \sigma_{n-1}+\int_{B\left(\xi_{2}, \epsilon_{2}\right) \backslash K\left(\theta_{0}\right)} v^{r} \mathrm{~d} \sigma_{n-1}-\int_{B\left(\xi_{1}, \epsilon_{1}\right) \cap K\left(\theta_{0}\right)} v^{r} \mathrm{~d} \sigma_{n-1} \\
& >\int_{K\left(\theta_{0}\right)} v^{r} \mathrm{~d} \sigma_{n-1},
\end{aligned}
$$

which contradicts the equation (3.7). The claim is therefore proved.

By the claim, $v^{r}$ is constant on $\partial K(\theta)$ for each $\theta$. Write $v^{r}(\theta)$ for that value. Additionally, $\left(v^{\#}\right)^{r}$ is constant on $\partial K(\theta)$ for each $\theta$ by definition, so write $\left(v^{\#}\right)^{r}(\theta)$ for that value. Since $J v=J v^{\#}$, we have

$$
\int_{K(\theta)} v^{r} \mathrm{~d} \sigma_{n-1}=\int_{K(\theta)}\left(v^{\#}\right)^{r} \mathrm{~d} \sigma_{n-1} .
$$

Using spherical coordinates, the above integral becomes

$$
\beta_{n-2} \int_{0}^{\theta} v^{r}(\theta) \sin ^{n-2} \theta \mathrm{d} \theta=\beta_{n-2} \int_{0}^{\theta}\left(v^{\#}\right)^{r}(\theta) \sin ^{n-2} \theta \mathrm{d} \theta,
$$

where $\beta_{n-2}=\sigma_{n-2}\left(\mathbb{S}^{n-2}\right)$. Differentiating the above equation with respect to $\theta$ implies $v^{r}(\theta)=\left(v^{\#}\right)^{r}(\theta)$. That is, $v=v^{\#}$.

Now let $f \in L^{2}(A)$ be a general function with mean zero and let $f_{k}$ and $v_{k}$ be as in Step 3 in the proof of Theorem 3.1. By the above, $v_{k}=v_{k}^{\#}$ on $A$. By passing to a subsequence of the original $f_{k}$ we may assume that $v_{k} \rightarrow v$ and $v_{k}^{\#} \rightarrow v^{\#}$ a.e. Hence $v=v^{\#}$ a.e.

Remark 3.6. Corollaries 3.4 and 3.5 have analogous statements on balls. However, since the remainder of this paper deals only with consequences of the shell results, we omit the statements of those results. 


\section{Consequences of main results}

\subsection{Comparison results on spheres and hemispheres}

In this section we develop consequences of Theorem 3.1 on spheres and hemispheres. Theorem 3.1 concerns cap symmetrization, a partial symmetrization. The corollaries below, on the other hand, deal with total symmetrizations, meaning the rearrangement takes place on the whole space rather than on submanifolds.

Spheres. We write $\Delta_{\mathbb{S}}$ and $\nabla_{\mathbb{S}}$ for the spherical Laplacian and spherical gradient on $\mathbb{S}^{n}$. We write $W^{1,2}\left(\mathbb{S}^{n}\right)$ for the Sobolev space of functions in $L^{2}\left(\mathbb{S}^{n}\right)$ that, once expressed in spherical coordinates, have weak partial derivatives in $L^{2}\left(\mathbb{S}^{n}\right)$.

Given $F \in L^{2}\left(\mathbb{S}^{n}\right)$, we say that $U \in W^{1,2}\left(\mathbb{S}^{n}\right)$ is a weak solution to

$$
-\Delta_{\mathbb{S}} U=F
$$

provided

$$
\int_{\mathbb{S}^{n}} \nabla_{\mathbb{S}} U \cdot \nabla_{\mathbb{S}} G \mathrm{~d} \sigma_{n}=\int_{\mathbb{S}^{n}} F G \mathrm{~d} \sigma_{n}
$$

for every $G \in W^{1,2}\left(\mathbb{S}^{n}\right)$.

We now have the following corollary to Theorem 3.1.

Corollary 4.1 (Spherical comparison theorem). Let $F \in L^{2}\left(\mathbb{S}^{n}\right)$ with $\int_{\mathbb{S}^{n}} F \mathrm{~d} \sigma_{n}=$ 0 . Assume $U$ and $V$ are weak solutions to

$$
-\Delta_{\mathbb{S}} U=F \text { in } \mathbb{S}^{n}, \quad-\Delta_{\mathbb{S}} V=F^{\#} \text { in } \mathbb{S}^{n},
$$

where $F^{\#}$ is the spherical rearrangement of $F$. Additionally assume $U$ and $V$ are additively normalized so that $\int_{\mathbb{S}^{n}} U \mathrm{~d} \sigma_{n}=\int_{\mathbb{S}^{n}} V \mathrm{~d} \sigma_{n}=0$. Then

$$
U^{\star} \leq V^{\star}
$$

on $(0, \pi)$. Thus for every convex function $\phi: \mathbb{R} \rightarrow \mathbb{R}$,

$$
\int_{\mathbb{S}^{n}} \phi(U) \mathrm{d} \sigma_{n} \leq \int_{\mathbb{S}^{n}} \phi(V) \mathrm{d} \sigma_{n} .
$$

Consequently,

$$
\begin{aligned}
\|U\|_{L^{p}\left(\mathbb{S}^{n}\right)} & \leq\|V\|_{L^{p}\left(\mathbb{S}^{n}\right)}, \quad 1 \leq p \leq \infty, \\
\underset{\mathbb{S}^{n}}{\operatorname{ess} u p} U & \leq \underset{\mathbb{S}^{n}}{\operatorname{ess} \sup } V, \\
\underset{\mathbb{S}^{n}}{\operatorname{ess}} U & \geq \underset{\mathbb{S}^{n}}{\operatorname{ess}} \inf V, \\
\underset{\mathbb{S}^{n}}{\operatorname{osc}} U & \leq \underset{\mathbb{S}^{n}}{\operatorname{osc}} V .
\end{aligned}
$$


Proof. The idea is to extend from the sphere to a spherical shell by homogeneity. Fix any $0<a<1<b<\infty$ and let $A=A(a, b)$ be the spherical shell in $\mathbb{R}^{n+1}$ with inner radius $a$ and outer radius $b$. Define functions $f, u: A \rightarrow \mathbb{R}$ by the homogeneity formulas

$$
\begin{aligned}
f(r \xi) & =\frac{1}{r^{2}} F(\xi), \\
u(r \xi) & =U(\xi),
\end{aligned}
$$

for $r \in(a, b)$ and $\xi \in \mathbb{S}^{n}$.

We first observe that $u$ solves

$$
\begin{aligned}
-\Delta u & =f \text { in } A \subset \mathbb{R}^{n+1}, \\
\frac{\partial u}{\partial n} & =0 \text { on } \quad \partial A,
\end{aligned}
$$

because $\Delta=\partial_{r r}+n r^{-1} \partial_{r}+r^{-2} \Delta_{\mathbb{S}}$. The normalization $\int_{A} u \mathrm{~d} x=0$ follows immediately from the definition of $u$, since $\int_{\mathbb{S}^{n}} U \mathrm{~d} \sigma_{n}=0$.

Define $v$ on $A$ by

$$
v(r \xi)=V(\xi)
$$

and observe that the cap symmetrization of $f$ is

$$
f^{\#}(r \xi)=\frac{1}{r^{2}} F^{\#}(\xi)
$$

Hence $v$ solves

$$
\begin{gathered}
-\Delta v=f^{\#} \text { in } A, \\
\frac{\partial v}{\partial n}=0 \text { on } \partial A,
\end{gathered}
$$

and $\int_{A} v \mathrm{~d} x=0$. Theorem 3.1 implies that for almost every $r \in(a, b)$ the inequality

$$
u^{\star}(r, \theta) \leq v^{\star}(r, \theta)
$$

holds for every $\theta \in(0, \pi)$. Pick any $r$ so that the above inequality holds. The definitions of $u$ and $v$ imply

$$
U^{\star}(\theta)=u^{\star}(r, \theta) \leq v^{\star}(r, \theta)=V^{\star}(\theta)
$$

for every $\theta \in(0, \pi)$ which gives the main conclusion of the corollary. The conclusions about convex means and so on now follow from Proposition 2.7 and Corollary 2.11 . 
Remark 4.2. In [15, Theorem 4.1], Cianchi obtains a similar comparison result to Corollary 4.1. Indeed, let $F, U$, and $V$ satisfy all the hypothesis of Corollary 4.1, but do not assume $U$ and $V$ have zero mean. Instead assume that the decreasing rearrangements $U^{*}(t)$ and $V^{*}(t)$ simultaneously vanish at some value of $t$ (in [15], $\left.t=\sigma_{n}\left(\left\{\xi \in \mathbb{S}^{n}: F(\xi) \geq 0\right\}\right)\right)$. Cianchi then obtains the inequalities $U_{+}^{*} \leq V_{+}^{*}$ and $U_{-}^{*} \leq V_{-}^{*}$ for the decreasing rearrangements of the positive and negative parts of $U$ and $V$. Cianchi's conclusion is stronger than that of Corollary 4.1, and the reason for that is the different normalization on the solutions $U$ and $V$. One can give a direct proof of Cianchi's result using the star function method as we now show.

Write

$$
\Delta_{\mathbb{S}}^{\star}=\frac{d^{2}}{d \theta^{2}}-(n-1) \cot \theta \frac{d}{d \theta}
$$

and

$$
\Delta_{\mathbb{S}}^{\star t}=\frac{d^{2}}{d \theta^{2}}-(n-1) \cot \theta \frac{d}{d \theta}-(n-1) \csc ^{2} \theta \cdot I,
$$

where $I$ is the identity. The operators $\Delta_{\mathbb{S}}^{\star}$ and $\Delta_{\mathbb{S}}^{\star t}$ act on functions that belong to $C^{2}((0, \pi))$. By [6, Theorem 5 and formula (5.9)], it follows that

$$
-\Delta_{\mathbb{S}}^{\star}\left(U^{\star}-V^{\star}\right) \leq F^{\star}-F^{\star}=0
$$

in the distributional sense. Spelled out, the above inequality reads

$$
-\int_{0}^{\pi}\left(U^{\star}(\theta)-V^{\star}(\theta)\right) \Delta_{\mathbb{S}}^{\star t} G(\theta) \mathrm{d} \theta \leq 0
$$

for all nonnegative test functions $G \in C_{c}^{\infty}((0, \pi))$. Now $U^{\#}$ and $V^{\#}$ are constant on cap boundaries $\partial K(\theta)$ for $0<\theta<\pi$. Write $U^{\#}(\theta)$ and $V^{\#}(\theta)$ for those values. Integrating by parts, (4.1) becomes

$$
\int_{0}^{\pi}\left(U^{\#}(\theta)-V^{\#}(\theta)\right) \frac{d}{d \theta}\left(\sin ^{n-1} \theta G(\theta)\right) \mathrm{d} \theta \leq 0
$$

which implies

$$
\int_{0}^{\pi}\left(U^{\#}(\theta)-V^{\#}(\theta)\right) G^{\prime}(\theta) \mathrm{d} \theta \leq 0
$$

for all nonnegative test functions $G \in C_{c}^{\infty}((0, \pi))$. An easy argument then gives $U^{\#}(\theta)-V^{\#}(\theta)$ is increasing in $\theta$, which implies $U^{*}(t)-V^{*}(t)$ is increasing in $t$. Hence, if $U^{*}(t)$ and $V^{*}(t)$ simultaneously vanish at some value of $t$, Cianchi's conclusion $U_{+}^{*} \leq V_{+}^{*}$ and $U_{-}^{*} \leq V_{-}^{*}$ then follows.

The following corollary tells us that the solution $V$ to the symmetrized problem of Corollary 4.1 is spherically rearranged.

Corollary 4.3. If $F$ and $V$ are as in Corollary 4.1, then $V=V^{\#}$ a.e.

Proof. Let $f$ and $v$ be obtained by homogeneity as in the proof of Corollary 4.1. By Corollary 3.5, $v=v^{\#}$ a.e. which implies $V=V^{\#}$ a.e. 
Hemispheres. We write $\mathbb{S}_{+}^{n}=\left\{\left(\xi_{1}, \xi_{2}, \ldots, \xi_{n+1}\right) \in \mathbb{S}^{n}: \xi_{n+1}>0\right\}$ for the upper hemisphere of $\mathbb{S}^{n}$. For a function $F \in L^{1}\left(\mathbb{S}_{+}^{n}\right)$, we extend $F$ to $\mathbb{S}^{n}$ by even reflection through the plane $\left(x_{n+1}=0\right)$. That is, we define $\tilde{F}: \mathbb{S}^{n} \rightarrow \mathbb{R}$ a.e. by

$$
\tilde{F}\left(\xi_{1}, \xi_{2}, \ldots, \xi_{n+1}\right)= \begin{cases}F\left(\xi_{1}, \xi_{2}, \ldots, \xi_{n+1}\right) & \text { if } \xi_{n+1}>0 \\ F\left(\xi_{1}, \xi_{2}, \ldots,-\xi_{n+1}\right) & \text { if } \xi_{n+1}<0 .\end{cases}
$$

We define the hemispherical rearrangement $F^{\#}: \mathbb{S}_{+}^{n} \rightarrow \mathbb{R}$ of the function $F$ by the formula

$$
F^{\#}\left(\xi_{1}, \xi_{2}, \ldots, \xi_{n+1}\right)=(\tilde{F})^{\#}\left(\xi_{1}, \xi_{2}, \ldots, \xi_{n+1}\right),
$$

where $(\tilde{F})^{\#}$ denotes the spherical rearrangement of $\tilde{F}$. A cautionary note: the above equation contains two \# symbols, but those symbols stand for different things. The \# on the left side is used to define the hemispherical rearrangement, whereas the \# on the right side means spherical rearrangement.

Write $\widetilde{F}^{\#}$ for the even reflection of the hemispherical rearrangement $F^{\#}$ through the plane $\left(x_{n+1}=0\right)$. We claim that

$$
\widetilde{F^{\#}}=(\tilde{F})^{\#}
$$

a.e. If $\xi \in \mathbb{S}_{+}^{n}$, then

$$
\widetilde{F}^{\#}(\xi)=F^{\#}(\xi)=(\tilde{F})^{\#}(\xi),
$$

where the first equality holds by the definition of even reflection, and the second equality holds by the definition of $F^{\#}$. On the other hand, if $\left(\xi_{1}, \xi_{2}, \ldots, \xi_{n+1}\right) \in \mathbb{S}^{n}$ and $\xi_{n+1}<0$, then

$$
\begin{aligned}
\widetilde{F}^{\#}\left(\xi_{1}, \xi_{2}, \ldots, \xi_{n+1}\right) & =F^{\#}\left(\xi_{1}, \xi_{2}, \ldots,-\xi_{n+1}\right) \\
& =(\tilde{F})^{\#}\left(\xi_{1}, \xi_{2}, \ldots,-\xi_{n+1}\right)=(\tilde{F})^{\#}\left(\xi_{1}, \xi_{2}, \ldots, \xi_{n+1}\right),
\end{aligned}
$$

where the first equality again holds by definition of even reflection and the second equality holds by the definition of $F^{\#}$. The third equality holds because $\left(\xi_{1}, \xi_{2}, \ldots, \xi_{n+1}\right)$ and $\left(\xi_{1}, \xi_{2}, \ldots,-\xi_{n+1}\right)$ have the same distance from $e_{1}$. Thus we have verified equation (4.3) for $\xi=\left(\xi_{1}, \xi_{2}, \ldots, \xi_{n+1}\right)$ so long as $\xi_{n+1} \neq 0$.

The star function of $F$, denoted by $F^{\star}$, is defined on the interval $(0, \pi)$ by the formula

$$
F^{\star}(\theta)=\max _{\sigma_{n}(E)=\sigma_{n}\left(K(\theta)_{+}\right)} \int_{E} F(\xi) \mathrm{d} \sigma_{n}(\xi),
$$

where the max is taken over all subsets $E \subset \mathbb{S}_{+}^{n}$ with the same surface measure as $K(\theta)_{+}$; we have written $K(\theta)_{+}=\left\{\left(\xi_{1}, \xi_{2}, \ldots, \xi_{n+1}\right) \in K(\theta): \xi_{n+1}>0\right\}$. As 
in Proposition 2.5, the max defining $F \star$ is achieved for some subset $E$, explaining our use of max rather than sup. As in Proposition 2.6, it follows that

$$
F^{\star}(\theta)=\int_{K(\theta)_{+}} F^{\#}(\xi) \mathrm{d} \sigma_{n}(\xi) .
$$

Before we state the next corollary, we need to discuss what it means to solve Poisson's equation on a hemisphere with Neumann boundary conditions.

We write $W^{1,2}\left(\mathbb{S}_{+}^{n}\right)$ for the Sobolev space of functions in $L^{2}\left(\mathbb{S}_{+}^{n}\right)$ that, once expressed in spherical coordinates, have weak partial derivatives that also belong to $L^{2}\left(\mathbb{S}_{+}^{n}\right)$. Given $F \in L^{2}\left(\mathbb{S}_{+}^{n}\right)$ we say a function $U \in W^{1,2}\left(\mathbb{S}_{+}^{n}\right)$ is a weak solution to

$$
\begin{aligned}
-\Delta_{\mathbb{S}} U & =F \text { in } \mathbb{S}_{+}^{n}, \\
\frac{\partial U}{\partial n} & =0 \text { on } \partial \mathbb{S}_{+}^{n},
\end{aligned}
$$

provided

$$
\int_{\mathbb{S}_{+}^{n}} \nabla_{\mathbb{S}} U \cdot \nabla_{\mathbb{S}} G \mathrm{~d} \sigma_{n}=\int_{\mathbb{S}_{+}^{n}} F G \mathrm{~d} \sigma_{n}
$$

for each $G \in W^{1,2}\left(\mathbb{S}_{+}^{n}\right)$.

We can now state and prove the hemispherical comparison result.

Corollary 4.4 (Hemisphere comparison theorem). Let $F \in L^{2}\left(\mathbb{S}_{+}^{n}\right)$ with $\int_{\mathbb{S}_{+}^{n}} F \mathrm{~d} \sigma_{n}=0$. Assume $U$ and $V$ are weak solutions to

$$
\begin{aligned}
-\Delta_{\mathbb{S}} U & =F \text { in } \mathbb{S}_{+}^{n}, & -\Delta_{\mathbb{S}} V & =F^{\#} \text { in } \mathbb{S}_{+}^{n}, \\
\frac{\partial U}{\partial n} & =0 \text { on } \partial \mathbb{S}_{+}^{n}, & \frac{\partial V}{\partial n} & =0 \text { on } \partial \mathbb{S}_{+}^{n} .
\end{aligned}
$$

Additionally assume $U$ and $V$ are additively normalized so that $\int_{\mathbb{S}_{+}^{n}} U \mathrm{~d} \sigma_{n}=$ $\int_{\mathbb{S}_{+}^{n}} V \mathrm{~d} \sigma_{n}=0$. Then

$$
U^{\star} \leq V^{\star}
$$

in $(0, \pi)$. Consequently, for every convex function $\phi: \mathbb{R} \rightarrow \mathbb{R}$ we have

$$
\int_{\mathbb{S}_{+}^{n}} \phi(U) \mathrm{d} \sigma_{n} \leq \int_{\mathbb{S}_{+}^{n}} \phi(V) \mathrm{d} \sigma_{n} .
$$

Additionally,

$$
\begin{aligned}
&\|U\|_{L^{p}\left(\mathbb{S}_{+}^{n}\right)} \leq\|V\|_{L^{p}\left(\mathbb{S}_{+}^{n}\right)}, \quad 1 \leq p \leq \infty, \\
& \underset{\mathbb{S}_{+}^{n}}{\operatorname{ess} \sup U} \leq \underset{\mathbb{S}_{+}^{n}}{\operatorname{ess} \sup V,} \\
& \underset{\mathbb{S}_{+}^{n}}{\operatorname{ess} \inf U} \geq \underset{\mathbb{S}_{+}^{n}}{\operatorname{ess} \inf V,} \\
& \quad \underset{\mathbb{S}_{+}^{n}}{\operatorname{osc}} U \leq \underset{\mathbb{S}_{+}^{n}}{\operatorname{osc} V .}
\end{aligned}
$$


Proof. First assume $F$ is Lipschitz continuous on $\overline{\mathbb{S}_{+}^{n}}$. Then $F^{\#}$ is also Lipschitz continuous on $\mathbb{S}_{+}^{n}$ (this follows from [6, proof of Theorem 3]). The solutions $U$ and $V$ then belong to $C^{2}\left(\overline{\mathbb{S}_{+}^{n}}\right)$ by [23, Theorem 3.2]. Extend $U, V$, and $F$ to $\mathbb{S}^{n}$ by reflection through the plane $\left(x_{n+1}=0\right)$ just as we did in equation (4.2) and denote these extensions by $\tilde{U}, \tilde{V}$, and $\tilde{F}$ respectively. Then $\tilde{U}$ and $\tilde{V}$ belong to $W^{1,2}\left(\mathbb{S}^{n}\right)$ since they have classically vanishing outer normals along the equator $\left(x_{n+1}=0\right)$.

We first claim that $\tilde{U}$ and $\tilde{V}$ solve

$$
-\Delta_{\mathbb{S}} \tilde{U}=\tilde{F} \text { in } \mathbb{S}^{n}, \quad-\Delta_{\mathbb{S}} \tilde{V}=(\tilde{F})^{\#} \text { in } \mathbb{S}^{n},
$$

together with the normalizations $\int_{\mathbb{S}^{n}} \tilde{U} \mathrm{~d} \sigma_{n}=\int_{\mathbb{S}^{n}} \tilde{V} \mathrm{~d} \sigma_{n}=0$.

The normalization follows immediately from the definition of $\tilde{U}$ and $\tilde{V}$ and since $\int_{\mathbb{S}_{+}^{n}} U \mathrm{~d} \sigma_{n}=\int_{\mathbb{S}_{+}^{n}} V \mathrm{~d} \sigma_{n}=0$.

To show (4.5) we appeal directly to the definition in equation (4.4). Let $G \in$ $W^{1,2}\left(\mathbb{S}^{n}\right)$ and define $\tilde{G}\left(\xi_{1}, \ldots, \xi_{n+1}\right)=G\left(\xi_{1}, \ldots,-\xi_{n+1}\right)$ for $\left(\xi_{1}, \ldots, \xi_{n+1}\right) \in$ $\mathbb{S}^{n}$. We compute

$$
\begin{aligned}
\int_{\mathbb{S}^{n}} \nabla_{\mathbb{S}} \tilde{U} \cdot \nabla_{\mathbb{S}} G \mathrm{~d} \sigma_{n} & =\int_{\mathbb{S}_{+}^{n}} \nabla_{\mathbb{S}} U \cdot \nabla_{\mathbb{S}} G \mathrm{~d} \sigma_{n}+\int_{\mathbb{S}_{+}^{n}} \nabla_{\mathbb{S}} U \cdot \nabla_{\mathbb{S}} \tilde{G} \mathrm{~d} \sigma_{n} \\
& =\int_{\mathbb{S}_{+}^{n}} F G \mathrm{~d} \sigma_{n}+\int_{\mathbb{S}_{+}^{n}} F \tilde{G} \mathrm{~d} \sigma_{n} \\
& =\int_{\mathbb{S}^{n}} \tilde{F} G \mathrm{~d} \sigma_{n} .
\end{aligned}
$$

Similarly, $-\Delta_{\mathbb{S}} \tilde{V}=\widetilde{F}^{\#}=(\tilde{F})^{\#}$ in $\mathbb{S}^{n}$ by (4.3). By Corollary 4.1, $\tilde{U}^{\star} \leq \tilde{V}^{\star}$ on $(0, \pi)$ which immediately implies $U^{\star} \leq V^{\star}$ on $(0, \pi)$. This gives the first conclusion of the theorem in the case where $F$ is Lipschitz continuous on $\overline{\mathbb{S}_{+}^{n}}$.

Now let $F \in L^{2}\left(\mathbb{S}_{+}^{n}\right)$ be a general function of mean zero and choose a sequence of test functions $F_{k} \in C_{c}^{\infty}\left(\mathbb{S}_{+}^{n}\right)$ with $F_{k} \rightarrow F$ in $L^{2}\left(\mathbb{S}_{+}^{n}\right)$. Let $U_{k}$ and $V_{k}$ solve

$$
\begin{aligned}
& -\Delta_{\mathbb{S}} U_{k}=F_{k} \text { in } \mathbb{S}_{+}^{n}, \quad-\Delta_{\mathbb{S}} V_{k}=F_{k}^{\#} \text { in } \mathbb{S}_{+}^{n}, \\
& \frac{\partial U_{k}}{\partial n}=0 \quad \text { on } \partial \mathbb{S}_{+}^{n}, \quad \frac{\partial V_{k}}{\partial n}=0 \quad \text { on } \partial \mathbb{S}_{+}^{n},
\end{aligned}
$$

and assume the $U_{k}$ and $V_{k}$ are normalized to have mean zero. The work above gives

$$
U_{k}^{\star} \leq V_{k}^{\star}
$$

on $(0, \pi)$ for every $k$.

As in the proof of Theorem 3.1, $U_{k} \rightarrow U$ and $V_{k} \rightarrow V$ in $L^{2}\left(\mathbb{S}_{+}^{n}\right)$. Since the spherical rearrangement is a contraction in the $L^{1}$ distance, $U_{k}^{\star} \rightarrow U^{\star}$ and $V_{k}^{\star} \rightarrow V^{\star}$ on $(0, \pi)$. Hence, letting $k \rightarrow \infty$ in $U_{k}^{\star} \leq V_{k}^{\star}$, we obtain $U^{\star} \leq V^{\star}$. The remaining inequalities about $L^{p}$ norms and so on follow from Proposition 2.7 and Corollary 2.11 . 
The following corollary tells us that the solution $V$ to the rearranged problem is hemispherically rearranged.

Corollary 4.5. Let $F$ and $V$ be as in Corollary 4.4. Then $V=V^{\#}$ a.e.

Proof. Let $\tilde{F}$ and $\tilde{V}$ be as in the proof of Corollary 4.4. By Corollary 4.3, $\tilde{V}=\tilde{V}^{\#}$ a.e. which implies $V=V^{\#}$ a.e.

\subsection{Kawohl's conjecture}

Kawohl raises the following conjecture in [19, page 61].

Conjecture 4.6 (Kawohl's conjecture). Let $R$ be the unit square $(0,1) \times(0,1)$ in $\mathbb{R}^{2}$ and $f: R \rightarrow \mathbb{R}$ a sufficiently smooth function with mean value zero. Consider the problems:

$$
\begin{array}{rlrlrl}
-\Delta u & =f \text { in } R, & -\Delta v & =f^{\#} \text { in } R, \\
\frac{\partial u}{\partial n} & =0 \text { on } \partial R, & \frac{\partial v}{\partial n} & =0 & \text { on } \partial R,
\end{array}
$$

where $f^{\#}$ is the monotone decreasing rearrangement of $f$ in the direction $y$.

Then the oscillation of $u$ over $R$ should be dominated by the oscillation of $v$.

To be precise, the monotone decreasing rearrangement of $f$ in the direction $y$ is defined in the following manner. Fix $x \in(0,1)$ and let $f^{x}:(0,1) \rightarrow \mathbb{R}$ denote the slice function $f^{x}(y)=f(x, y)$. Then $f^{\#}(x, y)=\left(f^{x}\right)^{*}(y)$ where $\left(f^{x}\right)^{*}$ is the decreasing rearrangement of the slice function $f^{x}$.

There are two keys to our proof of Kawohl's conjecture. The first of these is the two-dimensional version of Theorem 3.1. Now we discuss the second key, a rectangular comparison result obtained from Theorem 3.1 by a conformal mapping. A Jacobian factor is introduced from the conformal change of variables. We will see that this Jacobian factor interacts well with each of the rearrangements involved.

Before we prove an analogue of Theorem 3.1 in a square, we need to define the notion of a star function using the rearrangement Kawohl considered. Let $R=(0,1) \times(0,1)$. For $f \in L^{1}(R)$, we let $f^{\#}$ denote the monotone decreasing rearrangement of $f$ in the direction $y$. Let $f^{\star}: R \rightarrow \mathbb{R}$ be the star function defined by the formula

$$
f^{\star}(s, t)=\max _{|E|=t} \int_{E} f(s, \tau) \mathrm{d} \tau,
$$

where $s \in(0,1)$ and the max is taken over all measurable subsets $E \subseteq(0,1)$ of one-dimensional Lebesgue measure $t \in(0,1)$. Just as in Proposition 2.5, the max is achieved for some subset $E$, which explains our use of max instead of sup.

We now state the rectangular comparison result. We compare the solutions of two PDEs, one with given data and one with data rearranged monotonically in the $y$ direction. We see that the solution with rearranged data has a larger star function.

In the following result and in the remainder of the paper, we use $\mathrm{d} x$ and $\mathrm{d} m$ interchangeably for two-dimensional Lebesgue measure. 
Theorem 4.7 (Rectangular comparison theorem). Let $f \in L^{2}(R)$ where $\int_{R} f \mathrm{~d} m=0$ and suppose $u$ and $v$ are weak solutions to

$$
\begin{aligned}
-\Delta u & =f \text { in } R, & -\Delta v & =f^{\#} \text { in } R, \\
\frac{\partial u}{\partial n} & =0 \text { on } \partial R, & \frac{\partial v}{\partial n} & =0 \quad \text { on } \partial R,
\end{aligned}
$$

where $f^{\#}$ denotes the monotone decreasing rearrangement of $f$ in the direction $y$.

If $u$ and $v$ are additively normalized so that $\int_{R} u \mathrm{~d} m=\int_{R} v \mathrm{~d} m=0$, then for almost every $s \in(0,1)$,

$$
\int_{0}^{1} u(s, t) \mathrm{d} t=\int_{0}^{1} v(s, t) \mathrm{d} t
$$

and the inequality

$$
u^{\star}(s, t) \leq v^{\star}(s, t)
$$

holds for every $t \in(0,1)$. In particular, $u^{\star} \leq v^{\star}$ a.e. in $R$.

Proof.

Step 1: Conformally convert data $f$ and $f^{\#}$ on $R$ into data $g$ and $g^{\#}$ on an annulus $A$. First assume $f$ is Lipschitz continuous in $\bar{R}$ and let $A=A\left(1, e^{\pi}\right)=\{z \in$ $\left.\mathbb{C}: 1<|z|<e^{\pi}\right\}$. The exponential function $T(\zeta)=e^{\pi \zeta}$ maps $R$ conformally onto $A^{\star}$. Define

$$
g(z)=f\left(T^{-1}(z)\right)\left|\left(T^{-1}\right)^{\prime}(z)\right|^{2}=\frac{f\left(T^{-1}(z)\right)}{|\pi z|^{2}} \quad \text { for } z \in \overline{A^{\star}},
$$

where we choose the branch cut of $T^{-1}(z)=\frac{1}{\pi} \log z$ to lie along the negative imaginary axis. Notice $g$ is Lipschitz continuous on $\overline{A^{\star}}$. Extend $g$ to all of $\bar{A}$ by reflection across the real axis, that is, $g(z)=g(\bar{z})$. This extended function $g$ is Lipschitz continuous on $\bar{A}$. Since cap symmetrization is performed on circles and $|\pi z|^{2}$ is positive and constant on circles, it follows that

$$
g^{\#}(z)=\frac{f^{\#}\left(T^{-1}(z)\right)}{|\pi z|^{2}}
$$

for $z \in A^{\star}$. A cautionary note regarding the above equation: $g^{\#}$ denotes the cap symmetrization of $g$ whereas $f^{\#}$ is the monotone decreasing rearrangement of $f$ in the $y$ direction.

The normalization of $f$ implies that

$$
0=\int_{R} f(\zeta) \mathrm{d} m(\zeta)=\int_{A^{\star}} f\left(T^{-1}(z)\right)\left|\left(T^{-1}\right)^{\prime}(z)\right|^{2} \mathrm{~d} m(z)
$$


by a change of variable. Hence

$$
0=2 \int_{A^{\star}} g(z) \mathrm{d} m(z)=\int_{A} g(z) \mathrm{d} m(z) .
$$

This computation shows that $g$ satisfies the admissibility condition for input data into a Poisson equation with Neumann boundary conditions. It also explains why $g$ must include the Jacobian factor in its definition.

Let $U$ and $V$ solve

$$
\begin{array}{rlrl}
-\Delta U & =g \text { in } A, \quad-\Delta V & =g^{\#} \text { in } A, \\
\frac{\partial U}{\partial n} & =0 \text { on } \partial A, \quad \frac{\partial V}{\partial n}=0 \quad \text { on } \partial A,
\end{array}
$$

where $U$ and $V$ are normalized so that $\int_{A} U \mathrm{~d} m=\int_{A} V \mathrm{~d} m=0$. By [23, Theorem 3.2], $U$ and $V$ belong to $C^{2}(\bar{A})$.

Step 2: Obtain potential solutions $u$ and $v$ for the original problem. Define $u, v \in C^{2}(R) \cap C^{1}(\bar{R})$ by

$$
\begin{aligned}
& u=U \circ T, \\
& v=V \circ T .
\end{aligned}
$$

We calculate

$$
-\Delta u(\zeta)=-\Delta U(T(\zeta))\left|T^{\prime}(\zeta)\right|^{2}=g(T(\zeta))\left|T^{\prime}(\zeta)\right|^{2}=f(\zeta) .
$$

Similarly,

$$
-\Delta v(\zeta)=-\Delta V(T(\zeta))\left|T^{\prime}(\zeta)\right|^{2}=g^{\#}(T(\zeta))\left|T^{\prime}(\zeta)\right|^{2}=f^{\#}(\zeta),
$$

where the last equality follows from equality (4.6).

$T$ is conformal, hence takes arcs that are perpendicular to $\partial R$ into arcs that are perpendicular to $\partial A^{\star}$. Moreover, $T$ maps $\partial R$ onto $\partial A^{\star}$. It follows that $\frac{\partial u}{\partial n}=$ $\frac{\partial v}{\partial n}=0$ on $\partial R$.

We next show that $u$ and $v$ have the same mean over any vertical strip through $R$. To see this, we fix an $s \in(0,1)$ and compute the integral

$$
\begin{aligned}
\int_{0}^{1} u(s, t) \mathrm{d} t & =\int_{0}^{1} U\left(e^{\pi s} e^{i \pi t}\right) \mathrm{d} t \\
& =\frac{1}{2} \int_{-1}^{1} U\left(e^{\pi s} e^{i \pi t}\right) \mathrm{d} t \\
& =\frac{1}{2 \pi} \int_{-\pi}^{\pi} U\left(e^{\pi s} e^{i t}\right) \mathrm{d} t \\
& =\frac{1}{2 \pi} \int_{-\pi}^{\pi} V\left(e^{\pi s} e^{i t}\right) \mathrm{d} t \\
& =\int_{0}^{1} v(s, t) \mathrm{d} t,
\end{aligned}
$$


where the second to last equality follows from the first conclusion of Theorem 3.1. Now Theorem 3.1 implies $U^{\star} \leq V^{\star}$ on $A^{\star}$ and writing $\zeta=(s, t)$ we then calculate

$$
\begin{aligned}
u^{\star}(\zeta) & =\int_{0}^{t} u^{\#}(s, \tau) \mathrm{d} \tau=\frac{1}{2 \pi} \int_{-\pi t}^{\pi t} U^{\#}\left(e^{\pi s} e^{i \tau}\right) \mathrm{d} \tau=\frac{1}{2 \pi} U^{\star}(T(\zeta)) \\
& \leq \frac{1}{2 \pi} V^{\star}(T(\zeta))=v^{\star}(\zeta) .
\end{aligned}
$$

One last issue to resolve is that the hypothesis of Theorem 4.7 require that $u$ and $v$ have mean zero. Since $u$ and $v$ above have the same mean over each vertical segment, they certainly have the same mean over the rectangle $R$. Hence by subtracting that constant from $u$ and $v$ we can assume that both $u$ and $v$ have mean zero, and the conclusion $u^{\star} \leq v^{\star}$ will still hold.

Step 3: Approximation argument for arbitrary input data $f$. To complete the proof, one uses an approximation argument similar to the one used in the proof of Proposition 2.20. We omit the details.

Remark 4.8. We can reach the same conclusion in Theorem 4.7 if we only assume $u$ and $v$ have the same mean over the rectangle $R$, rather than assuming they both have mean zero.

We now prove Kawohl's conjecture.

Corollary 4.9 (Kawohl's conjecture). If $f$, $u$, and $v$ are as in Theorem 4.7, then

$$
\underset{R}{\operatorname{osc}} u \leq \underset{R}{\operatorname{osc}} v .
$$

Proof. By Theorem 4.7, for almost every $s \in(0,1)$, we have

$$
\int_{0}^{t} u^{\#}(s, \tau) \mathrm{d} \tau \leq \int_{0}^{t} v^{\#}(s, \tau) \mathrm{d} \tau
$$

for every $t \in(0,1)$. Additionally, $u$ and $v$ have the same mean over almost every vertical segment of $R$, hence Corollary 2.11 gives

$$
\underset{t \in(0,1)}{\operatorname{ess} \sup } u(s, t) \leq \underset{t \in(0,1)}{\operatorname{ess} \sup } v(s, t)
$$

and

$$
\underset{t \in(0,1)}{\operatorname{ess} \inf } v(s, t) \leq \underset{t \in(0,1)}{\operatorname{ess} i \inf } u(s, t) .
$$

Taking the ess sup over $s \in(0,1)$ in the first inequality and the ess inf over $s \in$ $(0,1)$ in the second inequality, we have

$$
\underset{R}{\operatorname{ess} \sup } u \leq \underset{R}{\operatorname{ess} \sup } v
$$


and

$$
\underset{R}{\operatorname{ess} \inf } v \leq \mathrm{ess} \inf _{R} u .
$$

Finally, if we combine the above ess sup and ess inf inequalities, we conclude

$$
\underset{R}{\operatorname{Osc}} u \leq \underset{R}{\operatorname{Oosc}} v
$$

as desired.

\section{Appendix}

\section{A. Star function inequalities}

In this appendix, we give a proof Baernstein's subharmonicity result, Theorem 2.20, the key tool in proving Theorem 3.1. All proofs in this appendix are those of Baernstein, and he has generously allowed me to present them here. This appendix has two sections. Section 1 contains a formula for the Laplacian used to establish the presubharmonicity and subharmonicity results in Section 2.

\section{A.1. A formula for the Laplacian}

Let $K: \mathbb{R}^{n} \rightarrow \mathbb{R}$ be a nonnegative radially decreasing smooth function supported in the unit ball satisfying the normalization $\int_{\mathbb{R}^{n}} K \mathrm{~d} x=1$. Set $K_{\epsilon}(x)=\epsilon^{-n} K\left(\epsilon^{-1} x\right)$ and let $*$ denote standard convolution in $\mathbb{R}^{n}$. Given a function $f \in L_{\text {loc }}^{1}(\Omega)$ with $\Omega \subseteq \mathbb{R}^{n}$ a domain, the convolution $f * K_{\epsilon}$ is defined on the set $\{x \in \Omega: d(x, \partial \Omega)>$ $\epsilon\}$.

Proposition A.1 (A formula for the Laplacian). For $u \in C^{2}(\Omega)$ and $K_{\epsilon}$ as above,

$$
\lim _{\epsilon \rightarrow 0} \frac{\left(K_{\epsilon} * u\right)(x)-u(x)}{C_{K} \epsilon^{2}}=\Delta u(x), \quad x \in \Omega,
$$

where $C_{K}=\frac{1}{2 n} \int_{\mathbb{B}^{n}}|x|^{2} K(x) \mathrm{d} x$. Moreover, convergence above holds locally uniformly in $\Omega$.

The above proposition is proved by examining the Taylor expansion of $u$ about the point $x$.

\section{A.2. Commutativity, presubharmonicity, and subharmonicity theorems in shells}

Let $A=A(a, b)=\left\{x \in \mathbb{R}^{n}: a<|x|<b\right\}$ be a spherical shell in $\mathbb{R}^{n}$ with inner radius $0 \leq a$ and outer radius $b<\infty$.

Our next theorem employs the following consequence of Baernstein's "Master inequality," and appears as [6, Corollary 4]. 
Theorem A.2 (Master inequality for cap symmetrization). Let $f, g: A \rightarrow \mathbb{R}$ and $K:[0, \infty) \rightarrow[0, \infty)$ be decreasing where $f\left(r \xi_{1}\right) g\left(r \xi_{2}\right) K\left(d\left(\xi_{1}, \xi_{2}\right)\right) \in$ $L^{1}\left(\mathbb{S}^{n-1} \times \mathbb{S}^{n-1}\right)$ for every $a<r<b$. Then,

$$
\int_{A \times A} f(x) g(y) K(|x-y|) \mathrm{d} x \mathrm{~d} y \leq \int_{A \times A} f^{\#}(x) g^{\#}(y) K(|x-y|) \mathrm{d} x \mathrm{~d} y,
$$

where $f^{\#}$ and $g^{\#}$ denote the cap symmetrizations of $f$ and $g$ respectively.

The following presubharmonicity result states that when $-\Delta u=f$, then in a very weak sense, $-\Delta u^{\#} \leq f^{\#}$.

Theorem A.3 (Presubharmonicity in Shells). Assume $u \in C^{2}(A)$ with $-\Delta u=$ $f$. Then the following inequality holds

$$
-\int_{A} u^{\#} \Delta g \mathrm{~d} x \leq \int_{A} f^{\#} g \mathrm{~d} x
$$

for each $g \in C_{c}^{2}(A)$ nonnegative with $g=g^{\#}$, where \# denotes the cap symmetrization of $g$.

Proof.

Step 1: Construction of $T^{r}$ and $h$. Writing $A=A(a, b)$, fix $a<r<b$. Define $T^{r}: \mathbb{S}^{n-1} \rightarrow\left[0, \sigma_{n-1}\left(\mathbb{S}^{n-1}\right)\right]$ by

$$
\begin{aligned}
T^{r}(\xi)= & \sigma_{n-1}\left(\left\{\zeta \in \mathbb{S}^{n-1}: u^{r}(\xi)<u^{r}(\zeta)\right\}\right) \\
& +\sigma_{n-1}\left(\left\{\zeta \in \mathbb{S}^{n-1}: u^{r}(\xi)=u^{r}(\zeta) \text { and } \psi(\xi)<\psi(\zeta)\right\}\right)
\end{aligned}
$$

where $\psi: \mathbb{S}^{n-1} \rightarrow \mathbb{R}$ denotes projection onto the first coordinate $\psi\left(\xi_{1}, \ldots, \xi_{n}\right)=$ $\xi_{1}$, and we have written $u^{r}$ for the slice function $u^{r}(\xi)=u(r \xi)$. Then $T^{r}$ is a measure-preserving transformation with

$$
u^{r}=\left(u^{r}\right)^{*} \circ T^{r} .
$$

Let $T: \mathbb{S}^{n-1} \rightarrow\left[0, \sigma_{n-1}\left(\mathbb{S}^{n-1}\right)\right]$ be the measure preserving transformation that takes a point $\xi \in \mathbb{S}^{n-1}$ to $\sigma_{n-1}(K(\theta))$ where $\theta$ is the angle $\xi$ makes with $e_{1}$. Then $T$ is the measure preserving transformation for which $F^{*} \circ T$ equals $F^{\#}$, the spherical rearrangement of $F$, for functions $F$ defined on $\mathbb{S}^{n-1}$. Since the functions $g^{r}$ are spherically rearranged, we have

$$
\left(g^{\#}\right)^{r}=g^{r}=\left(g^{r}\right)^{*} \circ T .
$$

Define a function $h$ on $A$ by

$$
h^{r}=\left(g^{r}\right)^{*} \circ T^{r}, \quad a<r<b .
$$


Then

$$
h^{\#}=g^{\#}=g .
$$

In particular, $h$ is compactly supported and bounded because $g$ has both of these properties. For fixed $a<r<b$, we have

$$
\begin{aligned}
\int_{\mathbb{S}^{n-1}} u^{r} h^{r} \mathrm{~d} \sigma_{n-1} & =\int_{\mathbb{S}^{n-1}}\left(\left(u^{r}\right)^{*} \circ T^{r}\right)\left(\left(g^{r}\right)^{*} \circ T^{r}\right) \mathrm{d} \sigma_{n-1} \\
& =\int_{0}^{\sigma_{n-1}\left(\mathbb{S}^{n-1}\right)}\left(u^{r}\right)^{*}\left(g^{r}\right)^{*} \mathrm{~d} t \\
& =\int_{\mathbb{S}^{n-1}}\left(\left(u^{r}\right)^{*} \circ T\right)\left(\left(g^{r}\right)^{*} \circ T\right) \mathrm{d} \sigma_{n-1} \\
& =\int_{\mathbb{S}^{n-1}}\left(u^{r}\right)^{\#}\left(g^{r}\right)^{\#} \mathrm{~d} \sigma_{n-1} \\
& =\int_{\mathbb{S}^{n-1}}\left(u^{r}\right)^{\#} g^{r} \mathrm{~d} \sigma_{n-1} .
\end{aligned}
$$

Multiplying the above equality through by $r^{n-1}$ and integrating from $r=a$ to $r=b$ gives

$$
\int_{A} u h \mathrm{~d} x=\int_{A} u^{\#} g \mathrm{~d} x
$$

Step 2: Convolution inequalities. We write $(\cdot, \cdot)$ for the standard $L^{2}$ inner product on $A$. Step 1 then shows

$$
(u, h)=\left(u^{\#}, g\right) .
$$

Let $K$ be a nonnegative symmetric bump function as in Section A.1. Then,

$$
\left(u, K_{\epsilon} * h\right)=\int_{A \times A} \epsilon^{-n} K_{0}\left(\epsilon^{-1}|y-x|\right) u(x) h(y) \mathrm{d} x \mathrm{~d} y,
$$

where we have written $K_{0}(r)=K\left(r e_{1}\right)$. Theorem A.2 gives

$$
\left(u, K_{\epsilon} * h\right) \leq\left(u^{\#}, K_{\epsilon} * h^{\#}\right)=\left(u^{\#}, K_{\epsilon} * g\right) .
$$

Thus,

$$
\left(K_{\epsilon} * u-u, h\right)=\left(u, K_{\epsilon} * h\right)-(u, h) \leq\left(u^{\#}, K_{\epsilon} * g-g\right),
$$

where the first equality holds because $K$ is radial. Dividing the above inequality through by $\epsilon^{2}$ and letting $\epsilon \rightarrow 0$, we conclude

$$
-\left(f^{\#}, g\right) \leq-(f, h) \leq\left(u^{\#}, \Delta g\right)
$$

where the first inequality follows from Hardy-Littlewood. 
We define a new domain $A^{\star} \subset \mathbb{R}^{2}$ to be the polar rectangle

$$
A^{\star}=\left\{(r, \theta) \in \mathbb{R}^{2}: a<r<b, \quad 0<\theta<\pi\right\} .
$$

Given $u \in L_{\text {loc }}^{1}(A)$, define a star function $u^{\star}: A^{\star} \rightarrow \mathbb{R}$ by

$$
u^{\star}(r, \theta)=\sup _{E} \int_{E} u(r \xi) \mathrm{d} \sigma_{n-1}(\xi),
$$

where the sup is taken over all measurable subsets $E \subseteq \mathbb{S}^{n-1}$ with surface measure $\sigma(E)=\sigma(K(\theta))$; we have written $K(\theta)$ for the spherical cap on $\mathbb{S}^{n-1}$ with center $e_{1}$ and spherical radius $\theta$. We then have

$$
u^{\star}(r, \theta)=\int_{K(\theta)} u^{\#}(r \xi) \mathrm{d} \sigma_{n-1}(\xi)
$$

where $u^{\#}$ is the cap symmetrization of $u$. Define an operator $J: L_{\text {loc }}^{1}(A, \mathrm{~d} x) \rightarrow$ $L_{\text {loc }}^{1}\left(A^{\star}, \mathrm{d} r \mathrm{~d} \theta\right)$ by

$$
J u(r, \theta)=\int_{K(\theta)} u(r \xi) \mathrm{d} \sigma_{n-1}(\xi) .
$$

Then

$$
u^{\star}=J u^{\#} \text { on } A^{\star} .
$$

We write $(\cdot, \cdot)$ for the standard inner product on $L^{2}(A, \mathrm{~d} x)$ and $(\cdot, \cdot) \star$ for the standard inner product on $L^{2}\left(A^{\star}, \mathrm{d} r \mathrm{~d} \theta\right)$. Define an operator $J^{t}: L_{\mathrm{loc}}^{1}\left(A^{\star}, \mathrm{d} r \mathrm{~d} \theta\right) \rightarrow$ $L_{\text {loc }}^{1}(A, \mathrm{~d} x)$ by

$$
J^{t} F(r \xi)=r^{1-n} \int_{\theta}^{\pi} F(r, t) \mathrm{d} t, \quad a<r<b, \quad \xi \in \mathbb{S}^{n-1},
$$

where $\theta$ is the angle that $\xi$ makes with $e_{1}$. One calculates that

$$
(J u, G)_{\star}=\left(u, J^{t} G\right),
$$

when say $u \in L_{\mathrm{loc}}^{1}(A)$ and $G \in L^{\infty}\left(A^{\star}\right)$ with compact support.

We define new operators $\Delta^{\star}$ and $\Delta^{\star t}$ which act on $C^{2}\left(A^{\star}\right)$ as follows:

$$
\begin{aligned}
\Delta^{\star} F= & \partial_{r r} F+\frac{n-1}{r} \partial_{r} F+r^{-2}\left[\partial_{\theta \theta} F-(n-2)(\cot \theta) \partial_{\theta} F\right], \\
\Delta^{\star t} F= & \partial_{r r} F-\frac{n-1}{r} \partial_{r} F+r^{-2}(n-1) F \\
& +r^{-2}\left[\partial_{\theta \theta} F+(n-2)(\cot \theta) \partial_{\theta} F-(n-2)\left(\csc ^{2} \theta\right) F\right] .
\end{aligned}
$$

Theorem A.4 below appears as in [6, equation (5.9)]. 
Theorem A.4 (Commutativity relation for cap symmetrization). If $u \in C^{2}(A)$, then

$$
J \Delta u=\Delta^{\star} J u
$$

on $A^{\star}$.

Before proceeding, we prove an adjoint version of the above result.

Theorem A.5 (Adjoint commutativity relation for cap symmetrization). If $G \in$ $C_{c}^{2}\left(A^{\star}\right)$, then

$$
J^{t} \Delta^{\star t} G=\Delta J^{t} G
$$

Proof. Given any $u \in C^{2}(A)$, we compute

$$
\begin{aligned}
\left(u, J^{t} \Delta^{\star t} G\right) & =\left(J u, \Delta^{\star t} G\right)_{\star}=\left(\Delta^{\star} J u, G\right)_{\star} \\
& =(J \Delta u, G)_{\star}=\left(\Delta u, J^{t} G\right)=\left(u, \Delta J^{t} G\right),
\end{aligned}
$$

where the third equality follows from Theorem A.4 and the fourth equality follows from Green's theorem. One then has via approximation that $\left(u, J^{t} \Delta^{\star t} G\right)=$ ( $u, \Delta J^{t} G$ ) for every function $u \in L^{2}(A)$, which gives the result.

Before proceeding, we make the following definition.

Definition A.6. For $u \in C^{2}(A)$ and $f \in L_{\text {loc }}^{1}(A)$, we say that $-\Delta^{\star} u^{\star} \leq f \star$ in the weak sense provided the inequality

$$
-\int_{A^{\star}} u^{\star} \Delta^{\star t} G \mathrm{~d} r \mathrm{~d} \theta \leq \int_{A^{\star}} f^{\star} G \mathrm{~d} r \mathrm{~d} \theta
$$

holds for every nonnegative $G \in C_{c}^{2}\left(A^{\star}\right)$.

Theorem A.7 (Subharmonicity for cap symmetrization). Suppose $u \in C^{2}(A)$ satisfies $-\Delta u=f$. Then

$$
-\Delta^{\star} u^{\star} \leq f^{\star}
$$

in the weak sense.

Proof. Letting $G \in C_{c}^{2}\left(A^{\star}\right)$ be nonnegative, we write $g=J^{t} G$ and compute

$$
\begin{aligned}
\left(u^{\star}, \Delta^{\star t} G\right)_{\star} & =\left(J u^{\#}, \Delta^{\star t} G\right)_{\star}=\left(u^{\#}, J^{t} \Delta^{\star t} G\right) \\
& =\left(u^{\#}, \Delta J^{t} G\right)=\left(u^{\#}, \Delta g\right),
\end{aligned}
$$


with the third equality following from Theorem A.5. Since $g=g^{\#}$ is nonnegative, Theorem A.3 gives

$$
-\left(u^{\#}, \Delta g\right) \leq\left(f^{\#}, g\right)
$$

Thus,

$$
-\left(u^{\star}, \Delta^{\star t} G\right)_{\star} \leq\left(f^{\#}, g\right)=\left(J f^{\#}, G\right)_{\star}=\left(f^{\star}, G\right)_{\star} .
$$

\section{References}

[1] A. Alvino, J. I. Diaz, P. L. Lions and G. Trombetti, Elliptic equations and Steiner symmetrization, Comm. Pure Appl. Math. 49 (1996), 217-236.

[2] A. Alvino, S. Matarasso and G. Trombetti, Elliptic boundary value problems: comparison results via symmetrization, Ricerche Mat. 51 (2002), 341-355.

[3] A. BAernstein II, Proof of Edrei's spread conjecture, Proc. London Math. Soc. (3) 26 (1973), 418-434.

[4] A. BAERNSTEIN II, Integral means, univalent functions and circular symmetrization, Acta Math. 133 (1974), 139-169.

[5] A. BAERNSTEIN II, How the *-function solves extremal problems, In: "Proceedings of the International Congress of Mathematicians", Helsinki, 1978, Acad. Sci. Fennica, Helsinki, 1980, 639-644.

[6] A. BAeRnsteIn II, A unified approach to symmetrization, In: "Partial Differential Equations of Elliptic Type", Cortona, 1992, 47-91, Sympos. Math., XXXV, Cambridge Univ. Press, Cambridge, 1994.

[7] A. BAERNSTEIN II, The *-function in complex analysis, In: "Geometric Function Theor", R. Kühnau (ed.), Handbook of Complex Analysis, Vol. I, Elsevier Science, 2002, 229-271.

[8] M. F. Betta, F. Brock, A. Mercaldo and M. R. Posteraro, A weighted isoperimetric inequality and applications to symmetrization, J. Inequal. Appl. 4 (1999), 21-240.

[9] M. F. Betta, F. Brock, A. Mercaldo and M. R. Posteraro, A comparison result related to Gauss measure, C. R. Math. Acad. Sci. Paris 334 (2002), 451-456.

[10] F. BROCK, Steiner symmetrization and periodic solutions of boundary value problems, $\mathrm{Z}$. Anal. Anwend. 13 (1994), 417-423.

[11] F. Brock, F. Chiacchio and A. Mercaldo, Weighted isoperimetric inequalities in cones and applications, Nonlinear Anal. 75 (2012), 5737-5755.

[12] F. CHIACCHIO, Steiner symmetrization for an elliptic problem with lower-order terms, Ricerche Matematiche 53 (2004), 87-106.

[13] F. CHIACCHIO, Comparison results for linear parabolic equations in unbounded domains via Gaussian symmetrization, Differential Integral Equations 17 (2004), 241-258.

[14] F. ChiACCHIO and V. M. MonetTi, Comparison results for solutions of elliptic problems via Steiner symmetrization, Differential Integral Equations 14 (2001), 1351-1366.

[15] A. CiAnCHI, Elliptic equations on manifolds and isoperimetric inequalities, Proc. Roy. Soc. Edinburgh Sect. A 114 (1990), 213-227.

[16] V. Ferone and A. Mercaldo, Neumann problems and Steiner symmetrization, In: "Comm. in Partial Differential Equations", Vol. 30, Issue 10, 2005, 1537-1553.

[17] A. Fryszkowski, "Fixed Point Theory for Decomposable Sets: Topological Fixed Point Theory and Its Applications", Kluwer Academic Publishers, Dordrecht, 2004.

[18] G. H. Hardy, J. Littlewood and G. Pólya, "Inequalities", Cambridge Mathematical Library, Cambridge University Press, Cambridge, 1988. 
[19] B. KawoHL, "Rearrangements and Convexity of Level Sets in PDE", Lecture Notes in Math., Vol. 1150, Springer, Berlin, 1985.

[20] E. H. LiEB and M. Loss, "Analysis", Second edition., Graduate Studies in Mathematics, Vol. 14, American Mathematical Society, Providence, RI, 2001.

[21] W. LitTMAn, A strong maximum principle for weakly L-subharmonic functions, J. Math. Mech. 8 (1959), 761-770.

[22] C. Maderna and S. SAlsa, Symmetrization in Neumann problems Appl. Anal. 9, (1979) 247-256.

[23] O. A. Ladyzenskaja and N. N. Ural'Ceva, "Linear and Quasilinear Elliptic Equations", Academic Press, New York-London, 1968.

[24] G. TAlenti, Elliptic equations and rearrangements, Ann. Scuola Norm. Sup. Pisa Cl. Sci. (4) 3 (1976), 697-718.

\author{
Bucknell University \\ Department of Mathematics \\ 374 Olin Science Building \\ Lewisburg, PA 17837, USA \\ Jeffrey.Langford@Bucknell.edu
}

